\title{
Synthesis and Characterization of Newly Fused 1,2-Dihydropyrido[3,4-b], Bridged Oxadiazol-2-yl, 4-Substituted-benzylidene Hydrazide and Arylidene 6-Chloroquinoxaline 1,4-Dioxides
}

\author{
Samy B. Said, ${ }^{* a}$ Fawzia Z. El-Ablack ${ }^{a}$ and Hani M. Elbeheiry ${ }^{a}$ \\ ${ }^{a}$ Department of Chemistry, Faculty of Science, Damietta University, 34517 New Damietta, Egypt
}

\begin{abstract}
Some simple reactions using commercially available chemicals were used in the preparation of new biologically remarkable quinoxaline 1,4-dioxide derivatives. Several steps performed on 4-chloro-2-nitroaniline resulted in a quinoxaline 1,4-dioxide derivative, which reacted with dimethylformamide dimethylacetal (DMF-DMA) to produce an enamine. Transamination of this enamine with anilines gave the fused 1,2-dihydropyrido[3,4- $b$ ] quinoxaline 5,10-dioxide derivatives via an addition-elimination mechanism. Basic condensation reaction of quinoxaline active methyl afforded unexpected decarboxylated arylidene derivatives by using different aromatic aldehydes. Bridged oxadiazol-2-yl derivatives were obtained from the reaction of a hydrazide derivative with carbon disulfide and $p$-nitrobenzoic acid respectively. Whereas, acidic condensation of that hydrazide with aromatic aldehydes afforded the arylidene hydrazides.
\end{abstract}

Keywords: quinoxaline 1,4-dioxide, transamination, enamine, arylidene, hydrazide

\section{Introduction}

Among the heterocyclic compounds, quinoxaline 1,4-dioxide (QdNO) and its derivatives proved an enormous scale of biological activities. These manifold biological properties include antimicrobial, antiprotozoal, antitumoral, antioxidant and anti-inflammatory activities. As antimicrobial, QdNOs can act as antibacterial, ${ }^{1,2}$ antimycobacterial ${ }^{3-5}$ and antifungal ${ }^{6,7}$ agents. Antitrypanosomal, ${ }^{8,9}$ antimalarial, ${ }^{10,11}$ antitrichomonas ${ }^{6,12}$ and antiamoebic ${ }^{13}$ activities are proved as types of the antiprotozoal activities of QdNOs. QdNOs are pivotal in the anticancer chemotherapy because they have a selective toxicity toward hypoxic cells. ${ }^{14-17}$ Also, antioxidant and anti-inflammatory activities have been studied in vitro and in vivo. ${ }^{18,19}$

There are two main strategies for the preparation of QdNOs. The first one, known as direct oxidation, is an old and non-specific method which produces a mixture of $N$-monoxides and $N, N$-dioxides. This method can be achieved via the oxidation of a quinoxaline derivative. Different oxidizing agents were used as $m$-chloroperoxybenzoic acid, ${ }^{20}$ peroxyformic acid ${ }^{21}$ and peroxyacetic acid. ${ }^{22}$

\footnotetext{
*e-mail: sbsaid@yahoo.com
}

The second strategy, called Beirut reaction, ${ }^{23}$ is a relatively new reaction and more commonly used. Depending on its ability to perform a cycloaddition reaction, benzofuroxan (1) (Figure 1) reacts with different reagents producing QdNOs. These different reagents include simple alkenes, ${ }^{24}$ simple ketones, ${ }^{25} \beta$-diketones, ${ }^{26,27}$ $\beta$-ketoesters, ${ }^{28} \beta$-ketoamides, ${ }^{29} \alpha$-enamines ${ }^{30}$ and malononitrile. ${ }^{31}$<smiles></smiles>

1<smiles>[O-][n+]1onc2ccc(Cl)cc21</smiles>

2
Figure 1. Benzofuroxan and 6-chlorobenzofuroxan.

Herein, we utilized Beirut reaction to synthesize some new 6-chloroquinoxaline 1,4-dioxide derivatives from the reaction of 6-chlorobenzofuroxan (2) (Figure 1) with ethyl acetoacetate. These compounds covers some 2-arylidene6-chloro-3-(ethoxycarbonyl)quinoxaline 1,4-dioxides, 8-chloro-1-oxo-2-substituted-1,2-dihydropyrido[3,4- $b$ ] quinoxaline 5,10-dioxide derivatives and some derivatives of 6-chloro-3-(hydrazinecarbonyl)-2-methylquinoxaline 1,4-dioxide. Biological activities of this set of compounds will be screened in a next study. 


\section{Results and Discussion}

This study describes the design, synthesis and characterization of some novel 6-chloroquinoxaline 1,4-dioxide derivatives. The starting material 6-chloro3-(ethoxycarbonyl)-2-methylquinoxaline 1,4-dioxide (7) was prepared in four steps as shown in Scheme 1. Diazotization of 4-chloro-2-nitroaniline (3) produced 1-chloro-2-(4-chloro-2-nitrophenyl)diazene (4), which further reacted with sodium azide to yield 1-azido-4-chloro2-nitrobenzene (5). ${ }^{15}$ Then under the effect of heat, the azide 5 gave off nitrogen gas forming 5-chlorobenzofuroxan (6) which tautomerizes in solutions to the major form 6-chlorobenzofuroxan (2). ${ }^{32}$ Ultimately and using Beirut reaction, we modified a previous method ${ }^{33}$ via reacting 2 with ethyl acetoacetate by using morpholine as a base instead of triethylamine. A promotion in yield from 26 to $42 \%$ was achieved by this modification to obtain the derivative 7 .

Due to the presence of the ester group and the $\mathrm{N}$-oxide bond in the quinoxaline (7), the methyl group at carbon-2 becomes activated and we exploited the active methyl group to prepare some novel quinoxaline derivatives via condensation reactions. The condensation of 7 with dimethylformamide dimethylacetal (DMF-DMA) gave (E)-6-chloro-2-(2-(dimethylamino)vinyl)3 -(ethoxycarbonyl)quinoxaline 1,4-dioxide (8) in an excellent yield (Scheme 2). From the ${ }^{1} \mathrm{H}$ nuclear magnetic resonance (NMR) data, the configuration of the olefinic double bond in the enamine $\mathbf{8}$ is trans. The two protons $\mathrm{H}_{\mathrm{a}}$ and $\mathrm{H}_{\mathrm{b}}$ have two doublet peaks of a chemical shift $(\delta) 4.7$ and $9.5 \mathrm{ppm}$, respectively, with a coupling constant $(J)$ of $12.5 \mathrm{~Hz}$. The low value of $J$ is due to the electronegative nitrogen atom, which withdraws electrons from the $\mathrm{C}-\mathrm{H}$ bond and weakens communication through the bonds. ${ }^{34}$ The high value of $\delta$ for $\mathrm{H}_{\mathrm{b}}$ is due to the anisotropic effect of the quinoxaline 1,4-dioxide scaffold. Furthermore, $\mathrm{H}_{\mathrm{b}}$ is shielded by the electron delocalization (mesomeric effect coming from the non bonding electrons of nitrogen atom) as shown in Scheme 3.

Transamination $^{35}$ of the enamine $\mathbf{8}$ with primary aromatic amines (aniline, $p$-toluidine and $p$-nitroaniline) in the presence of $p$-toluenesulfonic acid as a catalyst afforded the cyclized 8-chloro-1-oxo-2-substituted1,2-dihydropyrido[3,4-b]quinoxaline 5,10-dioxide derivatives (10a-c) (Scheme4). The 1,2-dihydropyrido[3,4-b] quinoxaline structures are confirmed from the spectral data where infrared (IR) spectra showed a shifting in the absorption of the carbonyl of the ester group from 1735 to $1670-1680 \mathrm{~cm}^{-1}$ due to the newly formed amide group.<smiles>Nc1ccc(Cl)cc1[N+](=O)[O-]</smiles>

3<smiles>O=[N+]([O-])c1ccc(Cl)cc1[N+](=O)[O-]</smiles>

4<smiles></smiles>

5<smiles></smiles>

Scheme 1.<smiles>CCOC(=O)c1c(C)[n+]([O-])c2ccc(Cl)cc2[n+]1[O-]</smiles>

7<smiles>[R20][R]([R20])([H])[C@H](I)CN(C)C(OC)OC</smiles>

Reflux, $12 \mathrm{~h}$<smiles>[2H]c1c(Cl)cc2c(c1C)[n+]([O-])c(C(=O)OCC)c(/C(C)=C(\C)N(C)C)[n+]2[O-]</smiles> 
<smiles>CCOC(=O)C(C)=C(C)C(C)=[N+](C)C</smiles>

Scheme 3.

Additionally, the disappearance of the ethyl group protons in the ${ }^{1} \mathrm{H}$ NMR and the drop of $J$ of the protons $\mathrm{H}_{\mathrm{a}}$ and $\mathrm{H}_{\mathrm{b}}$ from 12.5 to around $9.5 \mathrm{~Hz}$ (turned to cis configuration) confirmed the cyclization process.

The transamination mechanism was not clear and proceeded via the cis isomer and not the trans as mentioned in a previous work. ${ }^{36}$ We suggested that the formation of the cyclized product (10) took place via an addition-elimination mechanism. In order to support our point of view, the transamination was performed using a secondary amine (morpholine) and the produced morpholino derivative (9) have the trans configuration and the $J$ value of the protons $\mathrm{H}_{\mathrm{a}}$ and $\mathrm{H}_{\mathrm{b}}$ is $12.5 \mathrm{~Hz}$. From this observation, there are two possible products formed from the transamination reaction and the more stable one is produced as a major product. In case of the secondary amine, the major is the trans isomer, while the $c i s$ is the major in case of the primary amine. In fact, the formation of the pyrido derivatives $(\mathbf{1 0 a}-\mathbf{c})$ is accomplished via two sequential reactions. Transamination followed by intramolecular amidation get the task done. A plausible mechanism for the synthesis of $\mathbf{1 0}$ is shown in Scheme 5. The olefinic double bond in $\mathbf{8}$ abstract proton from $p$-toluenesulfonic acid to form $\mathbf{a}$, which is directly attacked by a primary amine to form $\mathbf{b}$. The newly formed intermediate $\mathbf{b}$ undergoes proton transfer followed by dimethylamine elimination to form $\mathbf{c}$ and a stereoisomer mixture of $\mathbf{d}$ and $\mathbf{e}$. Only the cis isomer e completes the amidation process through an intramolecular nucleophilic attack of the alkylated nitrogen on the carbonyl ester to form $\mathbf{f}$, which can eliminate a molecule of ethanol to form the cyclized product $\mathbf{1 0}$.

In the same manner, condensation of the quinoxaline 7 with different aromatic aldehydes gave the corresponding 2-arylidene-6-chloro-3-(ethoxycarbonyl)quinoxaline 1,4-dioxide (11) or the unexpected 2-arylidene6-chloroquinoxaline 1,4-dioxide (12) (Scheme 6). An excess amount of the aldehyde in the presence of piperidine as a catalyst was refluxed with the compound $\mathbf{7}$ for $2 \mathrm{~h}$ to obtain the desired arylidene derivative $\mathbf{1 1}$ or $\mathbf{1 2}$.

The structures of the obtained products (11a-f) were confirmed by their spectral data and elemental analysis. Thus, the ${ }^{1} \mathrm{H}$ NMR spectra showed that the vinylic protons $\left(\mathrm{H}_{\mathrm{a}}\right.$ and $\left.\mathrm{H}_{\mathrm{b}}\right)$ are also trans as the value of $J$ is around $16 \mathrm{~Hz}$. The IR spectra showed bands in the region of 1610 to $1587 \mathrm{~cm}^{-1}$ assignable to the double bonds in combination with aromatic absorptions. The mass spectrum of each compound manifested a molecular ion peak. The derivatives (12a-b) have the same previous spectra except that of the ester group. Protons $\mathrm{H}_{\mathrm{a}}, \mathrm{H} 2$, H4 and H6 in (12a) are shielded by the mesomeric effect as shown in Scheme 7. Also the $H_{D}$ proton is deshielded by the mesomeric effect in both $\mathbf{1 2} \mathbf{a}$ and $\mathbf{1 2 b}$, where it is shifted to $\delta 9.2$ and $9.16 \mathrm{ppm}$, respectively. The higher downfield hydrogen absorption in $\mathbf{1 2} \mathbf{b}$ at $\delta 10.42 \mathrm{ppm}$ is attributed to the hydroxyl group of the phenol moiety. This is because of the hydrogen bonding formation with one of the $N$-oxide group as shown in Scheme $7 \mathrm{~b}$. To explain this unexpected result, we focused on the aromatic aldehydes as the reaction conditions are the same. We

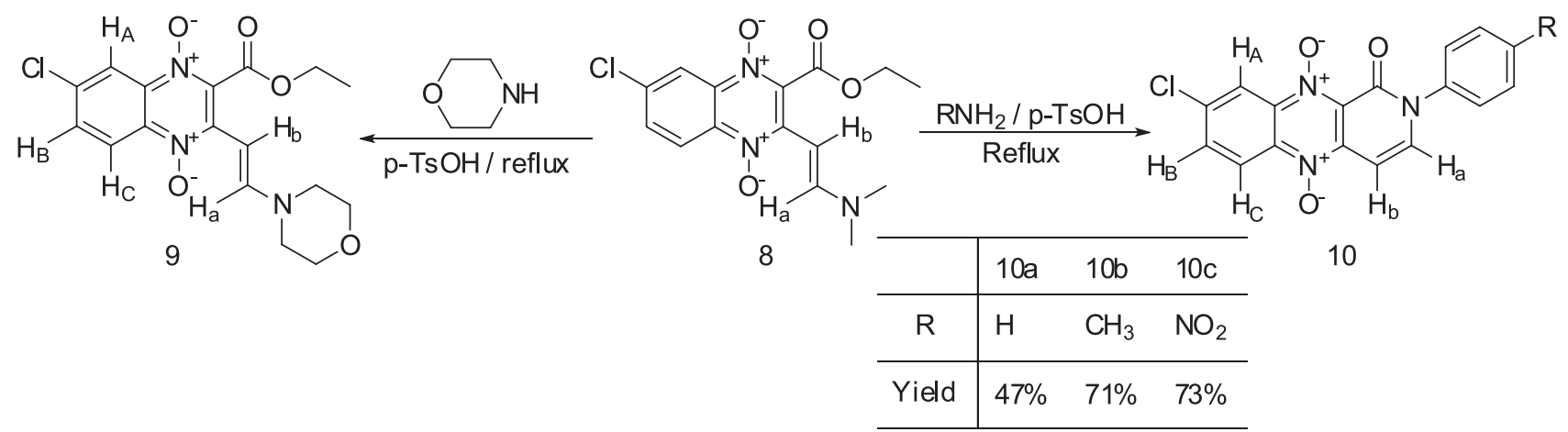



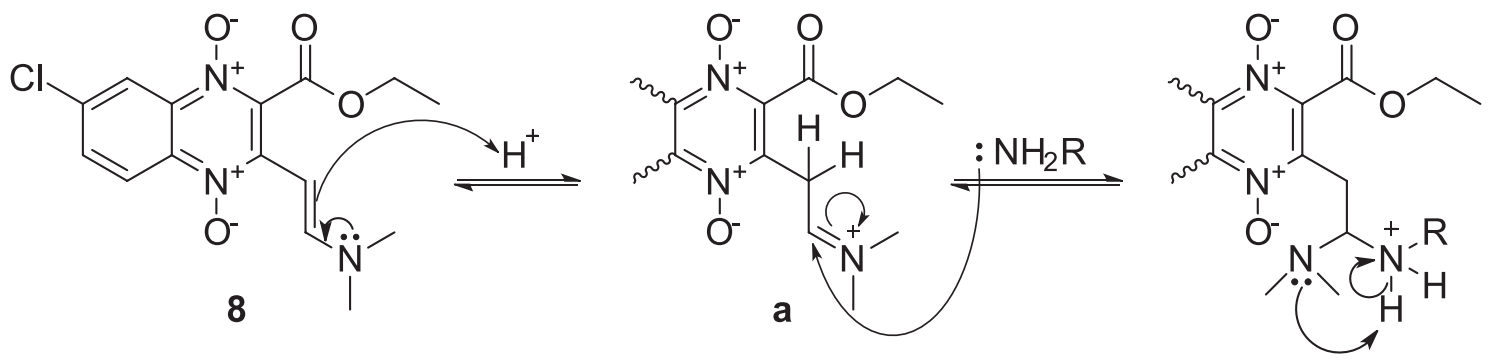<smiles>[R]N/C=C/c1c(C(=O)OCC)[n+]([O-])c(C)c(C)[n+]1[O-]</smiles>

d

$+$<smiles>CCOC(=O)c1c(C)c(C)c(C)[n+]([O-])c1C1=C(C)CCCC1</smiles>

$\mathrm{RHN}$

e

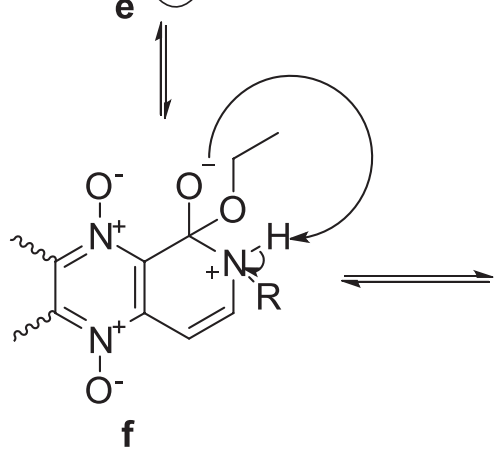

$\widehat{\mathrm{OH}}+$
$\stackrel{\mathrm{p}-\mathrm{TSO}}{\longleftarrow}$<smiles></smiles>

c

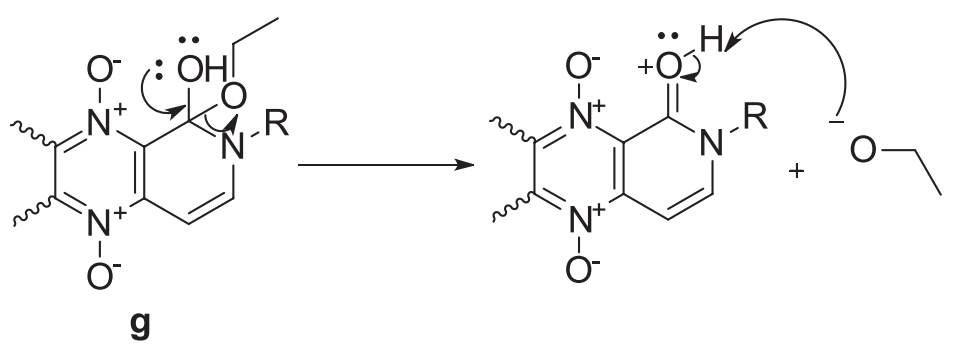<smiles>[R]n1ccc2c(c1=O)[n+]([O-])c1cc(Cl)ccc1[n+]2[O-]</smiles> 
<smiles>[R]c1ccc(C=Cc2ccc(C)cc2)cc1C(=O)OCC</smiles><smiles>[R]c1ccc(/C(C)=C(\C)c2c(C(=O)OCC)[n+]([O-])c3c([2H])c(Cl)c([Hg])c([CH])c3[n+]2[O-])cc1</smiles>

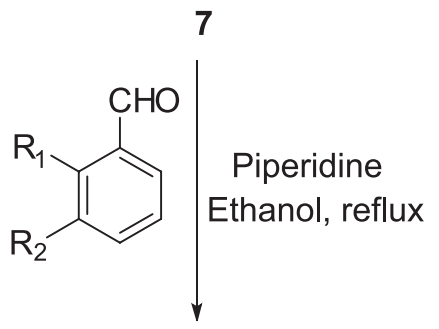

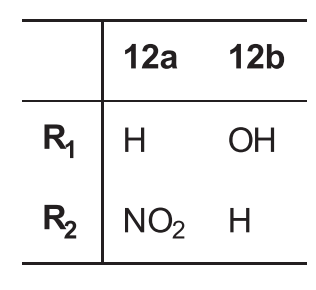

Scheme 6.<smiles>[R]c1cccc(/C(C)=C(\C)c2c(O)[n+]([O-])c3c([2H])c(Cl)c([2H])c(C)c3[n+]2[O-])c1[R]</smiles>

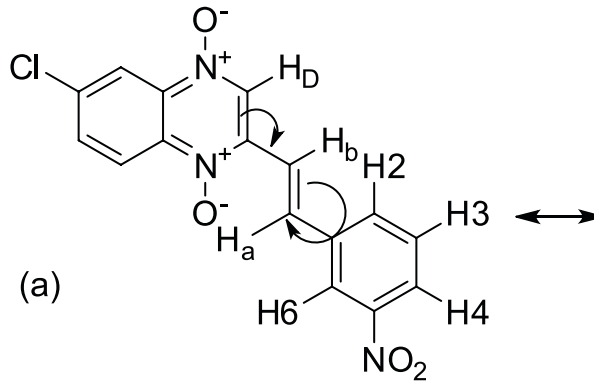

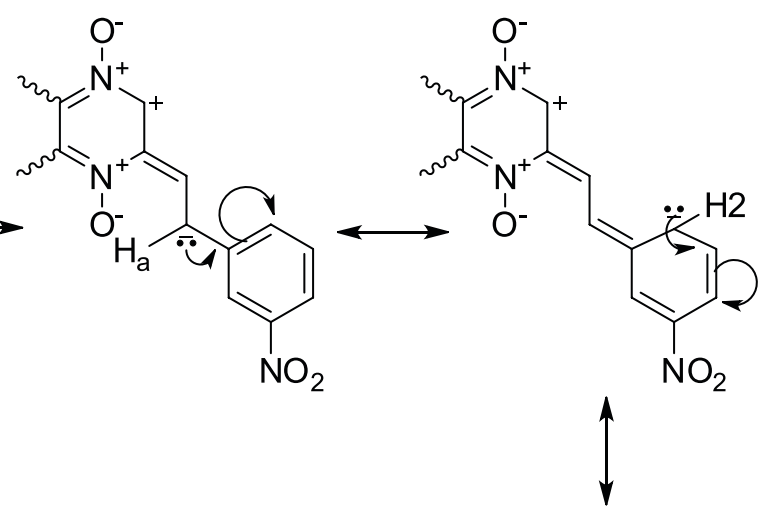<smiles>[O-][n+]1cc(/C=C/c2ccccc2O)[n+]([O-])c2ccc(Cl)cc21</smiles>

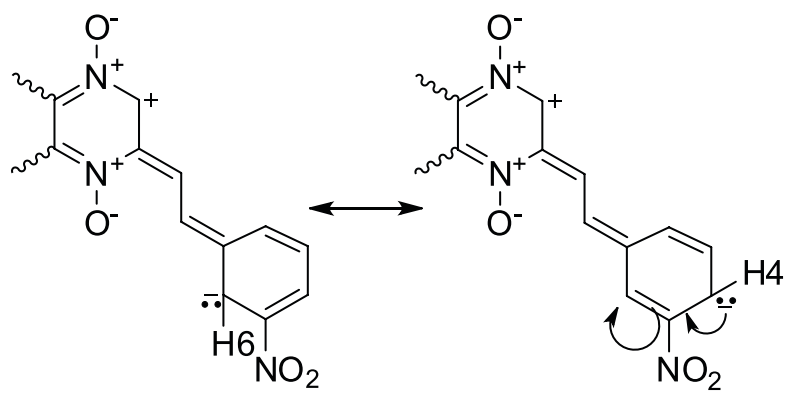

Scheme 7.

noted that in the case of using $m$-nitrobenzaldehyde or $o$-hydroxybenzaldehyde, the aldehyde group is activated toward the condensation reaction by the electron withdrawing nitro group or by the hydrogen bond of the hydroxyl group, respectively. Thus the condensation rate in these cases is faster than that of the other aldehydes.
Now, the formed water (by-product of the condensation), the basic medium, the high temperature and the time, are all perfect conditions for hydrolysis and decarboxylation of the ester group. This explanation was established by the complete characterization data of both 12a and $\mathbf{1 2 b}$, which prove the decarboxylation reaction. 
Heating of compound 7 with hydrazine hydrate under reflux on a steam bath for an hour afforded 6-chloro-3-(hydrazinecarbonyl)-2-methylquinoxaline 1,4-dioxide (13) in good yield (Scheme 8). The hydrazide 13 structure is confirmed by the spectral data and elemental analysis. The higher downfield hydrogen absorption in $\mathbf{1 3}$ at $\delta 9.97 \mathrm{ppm}$ is attributed to the NH group of the hydrazinecarbonyl moiety. This is because of the hydrogen bonding formation with one of the $N$-oxide group.

When the hydrazide $\mathbf{1 3}$ was refluxed for $24 \mathrm{~h}$ with excess carbon disulfide $\left(\mathrm{CS}_{2}\right)$ in ethanol as a solvent and potassium hydroxide as a base then acidified using (1 M) $\mathrm{HCl}$, 6-chloro-2-methyl-3-(5-thioxo-4,5-dihydro1,3,4-oxadiazol-2-yl)quinoxaline 1,4-dioxide (14) was produced in $68 \%$ yield (Scheme 9). Also when the hydrazide $\mathbf{1 3}$ was heated at $70{ }^{\circ} \mathrm{C}$ with $p$-nitrobenzoic acid in the presence of phosphorous oxychloride as a catalyst, the hydrazide group was converted into the 1,3,4-oxadiazole ring (Scheme 9). The produced 6-chloro2-methyl-3-(5-(4-nitrophenyl)-1,3,4-oxadiazol-2-yl) quinoxaline 1,4-dioxide (15) is confirmed by spectral data. Condensation of 13 with different aldehydes in the presence of acetic acid as a catalyst yielded the para substituted benzylidene hydrazide (16a-e) in very good yield
(Scheme 9). The derivatives 16a-e showed characteristic absorption peaks around 3300 and $1685 \mathrm{~cm}^{-1}$ for $\mathrm{N}-\mathrm{H}$ and carbonyl group, respectively. In ${ }^{1} \mathrm{H}$ NMR there is a peak of $\mathrm{N}-\mathrm{H}$ proton around $12 \mathrm{ppm}$, which exchanges with $\mathrm{D}_{2} \mathrm{O}$.

\section{Conclusions}

The synthesis of some new bioactive quinoxaline 1,4-dioxides was described. During our work we discovered that a basic condensation reaction can result in a hydrolysis recation followed by decarboxylation of an existing ester group. Also, the mechanism of transamination of enamines proved as an addition-elimination mechanism.

\section{Experimental}

All chemicals (AR graded) were commercially available and used without further purification. All liquid aldehydes were purified by extraction with a diluted solution of sodium carbonate, washed with water then dried over anhydrous sodium sulfate. Analytical thin-layer chromatography (TLC) was carried out using $0.2 \mathrm{~mm}$ precoated plates of silica gel G60 $\mathrm{F}_{254}$ (Merck). Visualization on TLC was achieved by UV light (254 and $366 \mathrm{~nm}$ ) or with an<smiles>CCOC(=O)c1c(C)[n+]([O-])c2ccc(Cl)cc2[n+]1[O-]</smiles>

7

13

Scheme 8.<smiles>[R]c1ccc(C=O)cc1</smiles><smiles>[R]c1ccc(/C([2H])=N/NC(=O)c2c(C)[n+]([O-])c3c([2H])c(C)c(Cl)cc3[n+]2[O-])cc1</smiles>

Scheme 9. 
iodine vapor. Plate chromatography was performed using precoated $20 \times 20 \mathrm{~cm}$ plates of silica gel $\mathrm{G}_{60} \mathrm{~F}_{254}$ (Merck). Melting points are uncorrected and were determined in an open capillary with a Griffin melting point apparatus. IR spectra were obtained for KBr pellets on JASCO 410 spectrometer with only selected absorptions being recorded in the range of 4000-400 $\mathrm{cm}^{-1}$. Ultraviolet absorption spectra were recorded in DMSO on a double beam JASCO V630 spectrophotometer in the wavelength range 300$900 \mathrm{~nm}$ in $1 \mathrm{~cm}$ quartz cuvettes. ${ }^{1} \mathrm{H}$ and ${ }^{13} \mathrm{C}$ NMR spectra were recorded on a Bruker Avance III $400 \mathrm{MHz}$ NMR spectrometer and Jeol ECA-II $500 \mathrm{MHz}$ NMR specrometer in $\mathrm{CDCl}_{3}$ and DMSO- $d_{6}$. Chemical shifts are expressed in $\delta$ ppm downfield from tetramethylsilane (TMS) as an internal reference. The mass spectra were recorded on Direct Inlet part to mass analyzer in Thermo Scientific GCMS model ISQ. Elemental analysis for C, H, and N was performed on FLASH 2000 CHNS/O analyzer, Thermo Scientific analyzer.

Synthesis of 6-chloro-3-(ethoxycarbonyl)-2-methylquinoxaline 1,4-dioxide (7)

To a solution of 4-chloro-2-nitroaniline 3 (8.63 g, $50 \mathrm{mmol})$ in water $(20 \mathrm{~mL})$ and concentrated $\mathrm{HCl}(12 \mathrm{~mL})$, a solution of $\mathrm{NaNO}_{2}(3.63 \mathrm{~g}, 52 \mathrm{mmol})$ in water $(13 \mathrm{~mL})$ was added dropwise at $0{ }^{\circ} \mathrm{C}$. After an hour of stirring, the formed yellow solution $\mathbf{4}$ was filtered off to remove the insoluble impurities. With stirring at $0{ }^{\circ} \mathrm{C}$, a solution of $\mathrm{NaN}_{3}(3.25 \mathrm{~g}, 50 \mathrm{mmol})$ in water $(13 \mathrm{~mL})$ was added dropwise to the previous solution. Immediately, the azide 5 began to precipitate, which was filtered off after finishing the addition. The azide 5 was refluxed in acetic acid $(50 \mathrm{~mL})$ till nitrogen gas ceased and was poured into crashed ice, the product 6 was filtered off, washed with cold water then it was dried. To a solution of $\mathbf{6}(1.70 \mathrm{~g}, 10 \mathrm{mmol})$ in ethyl acetoacetate $(1.30 \mathrm{~g}, 10 \mathrm{mmol})$ at $0{ }^{\circ} \mathrm{C}$, morpholine $(1.74 \mathrm{~g}$, $20 \mathrm{mmol}$ ) was added slowly. The ice bath was removed after an hour then the mixture was stirred for $12 \mathrm{~h}$ at room temperature in darkness. The produced precipitate was filtered off and washed with diethyl ether then recrystallized from methanol to form yellow needle-like crystals. Yield $1.2 \mathrm{~g}(42 \%) ; \mathrm{mp} 174-176{ }^{\circ} \mathrm{C}$; IR (KBr) v / cm 3093 (Ar-H), 2983 (C-H saturated), 1741 (C=O ester), 1599 (C=C aromatic), $1327(\mathrm{~N}-\mathrm{O}), 1276(\mathrm{C}-\mathrm{O}), 821(\mathrm{C}-\mathrm{Cl})$; UV (DMSO) $\lambda_{\max } / \mathrm{nm} 406 ;{ }^{1} \mathrm{H}$ NMR $\left(500 \mathrm{MHz}, \mathrm{DMSO}-d_{6}\right)$ $\delta 1.35\left(\mathrm{t}, J 7.2 \mathrm{~Hz}, 3 \mathrm{H}, \mathrm{OCH}_{2} \underline{\mathrm{CH}}_{3}\right), 2.42\left(\mathrm{~s}, 3 \mathrm{H}, \mathrm{CH}_{3}\right), 4.50$ $\left(\mathrm{q}, J 7.2 \mathrm{~Hz}, 2 \mathrm{H}, \mathrm{OCH}_{2} \mathrm{CH}_{3}\right), 8.02$ (dd, $J_{\text {ortho }} 9.2, J_{W} 2.3 \mathrm{~Hz}$, $\left.1 \mathrm{H}, \mathrm{H}_{\mathrm{B}}\right), 8.40\left(\mathrm{~d}, J_{W} 2.3 \mathrm{~Hz}, 1 \mathrm{H}, \mathrm{H}_{\mathrm{A}}\right), 8.46\left(\mathrm{~d}, J_{\text {ortho }} 9.2 \mathrm{~Hz}\right.$, $\left.1 \mathrm{H}, \mathrm{H}_{\mathrm{C}}\right) ;{ }^{13} \mathrm{C}$ NMR $\left(500 \mathrm{MHz}, \mathrm{DMSO}-d_{6}\right) \delta 13.80,14.13$, 63.39, 118.87, 122.07, 133.08, 135.56, 136.67, 136.98,
138.78, 159.46; MS (m/z) $282(60)[\mathrm{M}]^{+}, 284(20)[\mathrm{M}+2]^{+}$, 193 (100). Anal. calcd. for $\mathrm{C}_{12} \mathrm{H}_{11} \mathrm{ClN}_{2} \mathrm{O}_{4}$ : C, 50.99; H, 3.92; N, 9.91. Found: C, 50.79; H, 3.89; N, 9.93.

Synthesis of $(E)-6$-chloro-2-(2-(dimethylamino)vinyl)3-(ethoxycarbonyl)quinoxaline 1,4-dioxide (8)

A solution of 6-chloro-3-(ethoxycarbonyl)-2-methylquinoxaline 1,4-dioxide (7, $1.41 \mathrm{~g}, 5 \mathrm{mmol})$ and DMF-DMA $(0.90 \mathrm{~g}, 7.5 \mathrm{mmol})$ in dry benzene $(10 \mathrm{~mL})$ was refluxed for $12 \mathrm{~h}$. Solvent was evaporated under reduced pressure and the precipitate was titurated with diethyl ether to obtain solid product as red crystals. Yield $1.48 \mathrm{~g}(88 \%) ; \mathrm{mp}$ 162-163 ${ }^{\circ} \mathrm{C}$; IR (KBr) v / cm cm $^{-1} 3100(\mathrm{Ar}-\mathrm{H}), 2979(\mathrm{C}-\mathrm{H}$ saturated), 1737 ( $\mathrm{C}=\mathrm{O}$ ester $), 1616(\mathrm{C}=\mathrm{C}$ aliphatic $), 1591$ $(\mathrm{C}=\mathrm{C}$ aromatic), $1358(\mathrm{~N}-\mathrm{O}), 1282(\mathrm{C}-\mathrm{O}), 756(\mathrm{C}-\mathrm{Cl})$; $\mathrm{UV}$ (DMSO) $\lambda_{\text {max }} / \mathrm{nm} 370 ;{ }^{1} \mathrm{H} \mathrm{NMR}\left(500 \mathrm{MHz}, \mathrm{CDCl}_{3}\right.$ ) $\delta 1.45\left(\mathrm{t}, J 7.0 \mathrm{~Hz}, 3 \mathrm{H}, \mathrm{OCH}_{2} \underline{\mathrm{CH}}_{3}\right), 2.88\left(\mathrm{~s}, 3 \mathrm{H}, \mathrm{NCH}_{3}\right)$, 3.14 (s, 3H, $\underline{\mathrm{NCH}}_{3}$ ), 4.55 (q, J7.0 Hz, $\left.2 \mathrm{H}, \mathrm{OCH}_{2} \mathrm{CH}_{3}\right), 4.71$ (d, $J_{\text {trans }} 12.5 \mathrm{~Hz}, 1 \mathrm{H}, \mathrm{H}_{\mathrm{b}}$ ), 7.69 (dd, $J_{\text {ortho }} 9.2, J_{W} 2.2 \mathrm{~Hz}, 1 \mathrm{H}$, $\left.\mathrm{H}_{\mathrm{B}}\right), 8.38\left(\mathrm{~d}, J_{\text {ortho }} 9.2 \mathrm{~Hz}, 1 \mathrm{H}, \mathrm{H}_{\mathrm{C}}\right), 8.42\left(\mathrm{~d}, J_{W} 2.2 \mathrm{~Hz}, 1 \mathrm{H}\right.$, $\left.\mathrm{H}_{\mathrm{A}}\right), 9.53\left(\mathrm{~d}, J_{\text {trans }} 12.5 \mathrm{~Hz}, 1 \mathrm{H}, \mathrm{H}_{\mathrm{a}}\right) ;{ }^{13} \mathrm{C} \mathrm{NMR}(500 \mathrm{MHz}$, $\left.\mathrm{CDCl}_{3}\right) \delta 14.00,63.25,83.89,119.37,120.13,121.79$, 133.00, 133.03, 134.37, 136.61, 137.94, 151.69, 160.51; MS (m/z) $337(10)[\mathrm{M}]^{+}, 339$ (7) $[\mathrm{M}+2]^{+}, 73$ (100). Anal. calcd. for $\mathrm{C}_{15} \mathrm{H}_{16} \mathrm{ClN}_{3} \mathrm{O}_{4}$ : C, 53.34; H, 4.77; N, 12.44. Found: C, 53.44; H, 4.77; N, 12.70 .

Synthesis of (E)-6-chloro-3-(ethoxycarbonyl)2-(2-morpholinovinyl)quinoxaline 1,4-dioxide (9) and 8-chloro-1-oxo-2-substituted-1,2-dihydropyrido[3,4- $b$ ] quinoxaline 5,10-dioxide derivatives (10a-c)

A solution of (E)-6-chloro-2-(2-(dimethylamino)vinyl)3-(ethoxycarbonyl)quinoxaline 1,4-dioxide (8, $0.34 \mathrm{~g}$, $1 \mathrm{mmol}), p$-toluenesulfonic acid $(0.12 \mathrm{~g}, 1 \mathrm{mmol})$, the primary aromatic amine $(1 \mathrm{mmol})$, chloroform $(10 \mathrm{~mL})$ and isopropanol $(10 \mathrm{~mL})$ was refluxed for 4-8 h. The solvent was evaporated under reduced pressure and the precipitate formed was filtered off, washed with $\mathrm{Na}_{2} \mathrm{CO}_{3}$ solution $(50 \mathrm{~mL}, 30 \%)$, water $(50 \mathrm{~mL}), \mathrm{HCl}$ solution $(50 \mathrm{~mL}, 30 \%)$ then water $(50 \mathrm{~mL})$.

(E)-6-Chloro-3-(ethoxycarbonyl)-2-(2-morpholinovinyl) quinoxaline 1,4-dioxide (9)

Yield $0.26 \mathrm{~g}(68 \%)$; $\mathrm{mp} 156-158^{\circ} \mathrm{C}$; IR $(\mathrm{KBr}) \mathrm{v} / \mathrm{cm}^{-1}$ 3093 (Ar-H), 2985 (C-H saturated), 1743 (C=O ester), 1597 ( $\mathrm{C}=\mathrm{C}$ aliphatic), 1520 ( $\mathrm{C}=\mathrm{C}$ aromatic), $1362(\mathrm{~N}-\mathrm{O})$, 1230 (C-O), 764 (C-Cl); UV (DMSO) $\lambda_{\max } / \mathrm{nm} \mathrm{370;}$ ${ }^{1} \mathrm{H}$ NMR $\left(500 \mathrm{MHz}, \mathrm{CDCl}_{3}\right) \delta 1.45(\mathrm{t}, J 7.2 \mathrm{~Hz}, 3 \mathrm{H}$, $\left.\mathrm{OCH}_{2} \underline{\mathrm{CH}}_{3}\right), 3.34\left(\mathrm{t}, J 4.9 \mathrm{~Hz}, 4 \mathrm{H},\left(\mathrm{CH}_{2}\right)_{2} \mathrm{~N}\right), 3.75(\mathrm{t}$, 
$\left.J 4.9 \mathrm{~Hz}, 4 \mathrm{H},\left(\mathrm{CH}_{2}\right)_{2} \mathrm{O}\right), 4.56\left(\mathrm{q}, J 7.2 \mathrm{~Hz}, 2 \mathrm{H}, \mathrm{OCH}_{2} \mathrm{CH}_{3}\right)$, $4.72\left(\mathrm{~d}, J_{\text {trans }} 12.5 \mathrm{~Hz}, 1 \mathrm{H}, \mathrm{H}_{\mathrm{b}}\right), 7.69\left(\mathrm{dd}, J_{\text {ortho }} 9.1, J_{W} 2.3 \mathrm{~Hz}\right.$, $\left.1 \mathrm{H}, \mathrm{H}_{\mathrm{B}}\right), 8.39\left(\mathrm{~d}, J_{\text {ortho }} 9.1 \mathrm{~Hz}, 1 \mathrm{H}, \mathrm{H}_{\mathrm{C}}\right), 8.43\left(\mathrm{~m}, 1 \mathrm{H}, \mathrm{H}_{\mathrm{A}}\right)$, $9.53\left(\mathrm{~d}, J_{\text {trans }} 12.5 \mathrm{~Hz}, 1 \mathrm{H}, \mathrm{H}_{\mathrm{a}}\right) ;{ }^{13} \mathrm{C} \mathrm{NMR}\left(500 \mathrm{MHz}, \mathrm{CDCl}_{3}\right)$ $\delta 14.05,63.30,63.83,83.98,84.47,119.45,120.17,133.10$, 133.22, 134.47, 136.66, 138.03, 151.76, 160.56; MS (m/z) 379 (59) $[\mathrm{M}]^{+}, 381(25)[\mathrm{M}+2]^{+}, 362$ (100). Anal. calcd. for $\mathrm{C}_{17} \mathrm{H}_{18} \mathrm{ClN}_{3} \mathrm{O}_{5}$ : C, 53.76; H, 4.78; N, 11.06. Found: C, 53.79; H, 4.08; N, 11.01 .

8-Chloro-1-oxo-2-phenyl-1,2-dihydropyrido[3,4-b] quinoxaline 5,10-dioxide (10a)

Dark red crystals (methanol); yield $0.16 \mathrm{~g} \mathrm{(47 \% );} \mathrm{mp}$ 246-248 ${ }^{\circ} \mathrm{C}$; IR (KBr) v / $\mathrm{cm}^{-1} 3074(\mathrm{Ar}-\mathrm{H}), 1674(\mathrm{C}=\mathrm{O}$ amide), 1623 ( $\mathrm{C}=\mathrm{C}$ aliphatic), 1500 ( $\mathrm{C}=\mathrm{C}$ aromatic), 1312 (N-O), 1273 (C-O), 763 (C-Cl); UV (DMSO) $\lambda_{\max } / \mathrm{nm}$ 353; ${ }^{1} \mathrm{H}$ NMR (500 MHz, DMSO- $\left.d_{6}\right) \delta 7.46\left(\mathrm{~d}, J_{c i s} 8.0 \mathrm{~Hz}\right.$, $\left.1 \mathrm{H}, \mathrm{H}_{\mathrm{b}}\right), 7.49-7.62(\mathrm{~m}, 5 \mathrm{H}, \mathrm{Ar}), 7.82\left(\mathrm{dd}, J_{c i s} 8.0, J^{5} 4.7 \mathrm{~Hz}\right.$, $\left.1 \mathrm{H}, \mathrm{H}_{\mathrm{a}}\right), 7.99\left(\mathrm{dd}, J_{\text {ortho }} 9.3, J_{W} 2.3 \mathrm{~Hz}, 1 \mathrm{H}, \mathrm{H}_{\mathrm{B}}\right), 8.5\left(\mathrm{~d}, J_{\text {ortho }}\right.$ $\left.9.3 \mathrm{~Hz}, 1 \mathrm{H}, \mathrm{H}_{\mathrm{C}}\right), 8.52\left(\mathrm{~d}, J_{W} 2.3 \mathrm{~Hz}, 1 \mathrm{H}, \mathrm{H}_{\mathrm{A}}\right) ; \mathrm{MS}(\mathrm{m} / z)$ 339 (26) $[\mathrm{M}]^{+}, 341(11)[\mathrm{M}+2]^{+}, 323$ (100). Anal. calcd. for $\mathrm{C}_{17} \mathrm{H}_{10} \mathrm{ClN}_{3} \mathrm{O}_{3}$ : C, 60.10; H, 2.97; N, 12.37. Found: C, 59.97; H, 2.93; N, 12.25 .

8-Chloro-1-oxo-2-( $p$-tolyl)-1,2-dihydropyrido[3,4-b] quinoxaline 5,10 -dioxide (10b)

Dark red crystals (ethanol); yield $0.25 \mathrm{~g}(71 \%)$; $\mathrm{mp}>300{ }^{\circ} \mathrm{C}$; IR (KBr) v / cm ${ }^{-1} 3089(\mathrm{Ar}-\mathrm{H}), 2916(\mathrm{C}-\mathrm{H}$ saturated), $1676(\mathrm{C}=\mathrm{O}$ amide $), 1612(\mathrm{C}=\mathrm{C}$ aliphatic $), 1510$ $(\mathrm{C}=\mathrm{C}$ aromatic), $1315(\mathrm{~N}-\mathrm{O}), 1261(\mathrm{C}-\mathrm{O}), 819(\mathrm{C}-\mathrm{Cl})$; $\mathrm{UV}$ (DMSO) $\lambda_{\max } / \mathrm{nm} 353 ;{ }^{1} \mathrm{H}$ NMR (500 MHz, $\left.\mathrm{CDCl}_{3}\right) \delta 2.42$ (s, 3H, $\mathrm{CH}_{3}$ ), 7.29-7.33 (m, 4H, Ar), 7.48 (d, J $J_{\text {cis }} 8.0 \mathrm{~Hz}$, $\left.1 \mathrm{H}, \mathrm{H}_{\mathrm{b}}\right), 7.84\left(\mathrm{dd}, J_{\text {ortho }} 9.3, J_{W} 2.3 \mathrm{~Hz}, 1 \mathrm{H}, \mathrm{H}_{\mathrm{B}}\right), 8.56(\mathrm{~d}$, $\left.J_{\text {ortho }} 9.3 \mathrm{~Hz}, 1 \mathrm{H}, \mathrm{H}_{\mathrm{C}}\right), 8.60\left(\mathrm{~d}, J_{\text {cis }} 8.0 \mathrm{~Hz}, 1 \mathrm{H}, \mathrm{H}_{\mathrm{a}}\right), 8.68(\mathrm{~d}$, $\left.J_{W} 2.3 \mathrm{~Hz}, 1 \mathrm{H}, \mathrm{H}_{\mathrm{A}}\right) ;{ }^{13} \mathrm{C} \mathrm{NMR}\left(500 \mathrm{MHz}, \mathrm{CDCl}_{3}\right) \delta 21.22$, 95.27, 95.83, 120.16, 120.56, 121.75, 126.07, 126.37, 130.26, 130.46, 133.29, 134.79, 136.79, 138.95, 139.44, 154.82; MS (m/z) $353(13)[\mathrm{M}]^{+}, 306$ (100). Anal. calcd. for $\mathrm{C}_{18} \mathrm{H}_{12} \mathrm{ClN}_{3} \mathrm{O}_{3}: \mathrm{C}, 61.11 ; \mathrm{H}, 3.42 ; \mathrm{N}, 11.88$. Found: C, $60.95 ; \mathrm{H}, 3.13 ; \mathrm{N}, 12.20$.

8-Chloro-2-(4-nitrophenyl)-1-oxo-1,2-dihydropyrido[3,4-b] quinoxaline 5,10-dioxide (10c)

Dark red crystals (washed with boiling ethanol); yield $0.28 \mathrm{~g}(73 \%) ; \mathrm{mp} 264-265{ }^{\circ} \mathrm{C}$; IR $(\mathrm{KBr}) \vee / \mathrm{cm}^{-1} 3109$ (Ar-H), 1674 ( $\mathrm{C}=\mathrm{O}$ amide), 1631 ( $\mathrm{C}=\mathrm{C}$ aliphatic), 1529 $(\mathrm{C}=\mathrm{C}$ aromatic $), 1319(\mathrm{~N}-\mathrm{O}), 1269(\mathrm{C}-\mathrm{O}), 831(\mathrm{C}-\mathrm{Cl}) ; \mathrm{UV}$ (DMSO) $\lambda_{\max } / \mathrm{nm} 352 ;{ }^{1} \mathrm{H}$ NMR (500 MHz, DMSO- $d_{6}$ ) $\delta$ 7.83-8.03 (m, 4H, Ar), 8.06 (dd, $J_{\text {ortho }} 9.3, J_{W} 2.3 \mathrm{~Hz}, 1 \mathrm{H}$, $\left.\mathrm{H}_{\mathrm{B}}\right), 8.33-8.48\left(\mathrm{~m}, 3 \mathrm{H}, \mathrm{H}_{\mathrm{A}}, \mathrm{H}_{\mathrm{a}}, \mathrm{H}_{\mathrm{b}}\right), 8.53\left(\mathrm{~d}, J_{\text {ortho }} 9.3 \mathrm{~Hz}\right.$, $\left.1 \mathrm{H}, \mathrm{H}_{\mathrm{C}}\right) ; \mathrm{MS}(\mathrm{m} / z) 384(18)[\mathrm{M}]^{+}, 386(2)[\mathrm{M}+2]^{+}, 383$
(100). Anal. calcd. for $\mathrm{C}_{17} \mathrm{H}_{9} \mathrm{ClN}_{4} \mathrm{O}_{5}: \mathrm{C}, 53.07 ; \mathrm{H}, 2.36 ; \mathrm{N}$, 14.56. Found: C, 53.39; H, 2.14; N, 14.94 .

Synthesis of 6-chloro-2-arylidene-3-(ethoxycarbonyl) quinoxaline 1,4-dioxide (11a-f) and 6-chloro-2-arylidenequinoxaline 1,4-dioxide (12a-b)

To a solution of 6-chloro-3-(ethoxycarbonyl)2-methylquinoxaline 1,4-dioxide (7, $0.28 \mathrm{~g}, 1 \mathrm{mmol})$ and the corresponding aromatic aldehyde $(3 \mathrm{mmol})$ in ethanol $(2 \mathrm{~mL})$, two drops of piperidine were added as a basic catalyst. The reaction mixture was heated under reflux for $2 \mathrm{~h}$ in an oil bath at $110^{\circ} \mathrm{C}$, left to cool then ethanol $(3 \mathrm{~mL})$ was added. The obtained precipitate was filtered off, washed with diethyl ether, purified via plate chromatography and recrystalized from the proper solvent.

(E)-6-Chloro-3-(ethoxycarbonyl)-2-styrylquinoxaline 1,4-dioxide (11a)

Fluffy yellowish orange solid (methanol); yield $0.10 \mathrm{~g}$ (27\%); mp 198-200 ${ }^{\circ} \mathrm{C}$; IR (KBr) v / cm ${ }^{-1} 3081(\mathrm{Ar}-\mathrm{H})$, 2979 (C-H saturated), 1733 (C=O ester), $1606(\mathrm{C}=\mathrm{C}$ aliphatic), $1498(\mathrm{C}=\mathrm{C}$ aromatic), $1360(\mathrm{~N}-\mathrm{O}), 1238$ (C-O), 754 (C-Cl); UV (DMSO) $\lambda_{\max } / \mathrm{nm} 330 ;{ }^{1} \mathrm{H}$ NMR $\left(500 \mathrm{MHz}, \mathrm{CDCl}_{3}\right) \delta 1.44$ (t, J 7.2 Hz, 3H, $\mathrm{OCH}_{2} \mathrm{CH}_{3}$ ), 4.59 (q, $J 7.2 \mathrm{~Hz}, 2 \mathrm{H}, \mathrm{OCH}_{2} \mathrm{CH}_{3}$ ), 7.07 (d, $J_{\text {trans }} 16.5 \mathrm{~Hz}$, $\left.1 \mathrm{H}, \mathrm{H}_{\mathrm{b}}\right), 7.40(\mathrm{~m}, 3 \mathrm{H}, \mathrm{Ar}), 7.55$ (dd, $J_{\text {ortho }} 7.5, J_{W} 2.3 \mathrm{~Hz}$, $2 \mathrm{H}, \mathrm{Ar}), 7.80\left(\mathrm{dd}, J_{\text {ortho }} 9.0, J_{W} 2.3 \mathrm{~Hz}, 1 \mathrm{H}, \mathrm{H}_{\mathrm{B}}\right), 8.35(\mathrm{~d}$, $\left.J_{\text {trans }} 16.5 \mathrm{~Hz}, 1 \mathrm{H}, \mathrm{H}_{\mathrm{a}}\right), 8.56\left(\mathrm{~m}, 2 \mathrm{H}, \mathrm{H}_{\mathrm{A}}, \mathrm{H}_{\mathrm{C}}\right) ;{ }^{13} \mathrm{C} \mathrm{NMR}$ $\left(500 \mathrm{MHz}, \mathrm{CDCl}_{3}\right) \delta 14.06,63.96,114.81,119.92$, $122.99,128.93,129.35,133.59,134.19,135.74,136.21$, 136.32, 136.86, 136.89, 138.71, 140.05, 159.63; MS ( $/ \mathrm{m} / \mathrm{z})$ 370 (17) $[\mathrm{M}]^{+}, 105$ (100). Anal. calcd. for $\mathrm{C}_{19} \mathrm{H}_{15} \mathrm{ClN}_{2} \mathrm{O}_{4}$ : C, 61.55; H, 4.08; N, 7.56. Found: C, 61.78; H, 3.92; N, 7.72 .

(E)-6-Chloro-2-(4-chlorostyryl)-3-(ethoxycarbonyl) quinoxaline 1,4-dioxide (11b)

Fluffy yellow solid; yield $0.12 \mathrm{~g}(30 \%)$; $\mathrm{mp} 191-192{ }^{\circ} \mathrm{C}$; $\operatorname{IR}(\mathrm{KBr}) v / \mathrm{cm}^{-1} 3100$ (Ar-H), 2983 (C-H saturated), 1739 ( $\mathrm{C}=\mathrm{O}$ ester), 1606 ( $\mathrm{C}=\mathrm{C}$ aliphatic), $1506(\mathrm{C}=\mathrm{C}$ aromatic $)$, 1361 (N-O), 1274 (C-O), 806 (C-Cl); UV (DMSO) $\lambda_{\max } / \mathrm{nm} 333 ;{ }^{1} \mathrm{H}$ NMR $\left(500 \mathrm{MHz}, \mathrm{CDCl}_{3}\right) \delta 1.38(\mathrm{t}$, $\left.J 7.2 \mathrm{~Hz}, 3 \mathrm{H}, \mathrm{OCH}_{2} \mathrm{CH}_{3}\right), 4.53\left(\mathrm{q}, J 7.2 \mathrm{~Hz}, 2 \mathrm{H}, \mathrm{OCH}_{2} \mathrm{CH}_{3}\right)$, $6.95\left(\mathrm{~d}, J_{\text {trans }} 16.3 \mathrm{~Hz}, 1 \mathrm{H}, \mathrm{H}_{\mathrm{b}}\right), 7.31$ (d, $\left.J_{\text {ortho }} 8.4 \mathrm{~Hz}, 2 \mathrm{H}, \mathrm{Ar}\right)$, $7.41\left(\mathrm{~d}, J_{\text {ortho }} 8.4 \mathrm{~Hz}, 2 \mathrm{H}, \mathrm{Ar}\right), 7.75\left(\mathrm{dd}, J_{\text {ortho }} 9.3, J_{W} 2 \mathrm{~Hz}\right.$, $\left.1 \mathrm{H}, \mathrm{H}_{\mathrm{B}}\right), 8.27\left(\mathrm{~d}, J_{\text {trans }} 16.3 \mathrm{~Hz}, 1 \mathrm{H}, \mathrm{H}_{\mathrm{a}}\right), 8.50\left(\mathrm{~m}, 2 \mathrm{H}, \mathrm{H}_{\mathrm{A}}\right.$, $\left.\mathrm{H}_{\mathrm{C}}\right) ;{ }^{13} \mathrm{C}$ NMR $\left(500 \mathrm{MHz}, \mathrm{CDCl}_{3}\right) \delta 14.03,63.92,114.28$, 119.86, 121.96, 127.82, 128.95, 129.05, 130.28, 133.55, 135.64, 136.21, 136.85, 137.17, 138.56, 141.56, 159.66; MS $(\mathrm{m} / \mathrm{z}) 404(86)[\mathrm{M}]^{+}, 406(53)[\mathrm{M}+2]^{+}, 139(100)$. 
Anal. calcd. for $\mathrm{C}_{19} \mathrm{H}_{14} \mathrm{Cl}_{2} \mathrm{~N}_{2} \mathrm{O}_{4}$ : C, 56.32; $\mathrm{H}, 3.48 ; \mathrm{N}, 6.91$. Found: C, 56.42; H, 3.29; N, 6.91.

(E)-6-Chloro-2-(4-(dimethylamino)styryl)-3-(ethoxycarbonyl)quinoxaline 1,4-dioxide (11c)

Dark red solid; yield $0.12 \mathrm{~g}(29 \%)$; mp 218-220 ${ }^{\circ} \mathrm{C}$; IR $(\mathrm{KBr}) v / \mathrm{cm}^{-1} 3074(\mathrm{Ar}-\mathrm{H}), 2965$ (C-H saturated), 1743 $(\mathrm{C}=\mathrm{O}$ ester $), 1625(\mathrm{C}=\mathrm{C}$ aliphatic $), 1587(\mathrm{C}=\mathrm{C}$ aromatic $)$, 1357 (N-O), 1269 (C-O), 798 (C-Cl); UV (DMSO) $\lambda_{\max } / \mathrm{nm} \mathrm{403;}{ }^{1} \mathrm{H}$ NMR $\left(400 \mathrm{MHz}, \mathrm{CDCl}_{3}\right) \delta 1.50(\mathrm{t}$, $\left.\left.J 7.0 \mathrm{~Hz}, 3 \mathrm{H}, \mathrm{OCH}_{2} \mathrm{CH}_{3}\right), 3.08\left(\mathrm{~s}, 6 \mathrm{H}, \mathrm{N}_{\left(\mathrm{CH}_{3}\right.}\right)_{2}\right), 4.64$ (q, $\left.J 7.0 \mathrm{~Hz}, 2 \mathrm{H}, \mathrm{OCH}_{2} \mathrm{CH}_{3}\right), 6.72\left(\mathrm{~d}, J_{\text {ortho }} 8.5 \mathrm{~Hz}, 2 \mathrm{H}, \mathrm{Ar}\right), 6.87$ $\left(\mathrm{d}, J_{\text {trans }} 16.0 \mathrm{~Hz}, 1 \mathrm{H}, \mathrm{H}_{\mathrm{b}}\right), 7.49\left(\mathrm{~d}, J_{\text {ortho }} 8.5 \mathrm{~Hz}, 2 \mathrm{H}, \mathrm{Ar}\right), 7.81$ $\left(\mathrm{dd}, J_{\text {ortho }} 9.2, J_{W} 2.2 \mathrm{~Hz}, 1 \mathrm{H}, \mathrm{H}_{\mathrm{B}}\right), 8.53\left(\mathrm{~d}, J_{\text {trans }} 16.0 \mathrm{~Hz}, 1 \mathrm{H}\right.$, $\left.\mathrm{H}_{\mathrm{a}}\right), 8.58\left(\mathrm{~m}, 2 \mathrm{H}, \mathrm{H}_{\mathrm{A}}, \mathrm{H}_{\mathrm{C}}\right) ;{ }^{13} \mathrm{C} \mathrm{NMR}\left(400 \mathrm{MHz}, \mathrm{CDCl}_{3}\right)$ $\delta$ 14.07, 29.68, 40.13, 63.73, 108.69, 111.97, 119.71, $119.75,121.69,126.41,129.75,133.34,142.40,151.83$, 151.87, 160.00; MS (m/z) $413(16)[\mathrm{M}]^{+}, 415(6)[\mathrm{M}+2]^{+}$, 397 (100). Anal. calcd. for $\mathrm{C}_{21} \mathrm{H}_{20} \mathrm{ClN}_{3} \mathrm{O}_{4}: \mathrm{C}, 60.95 ; \mathrm{H}, 4.87$; N, 10.15. Found: C, 60.93; H, 4.84; N, 10.13 .

(E)-6-Chloro-3-(ethoxycarbonyl)-2-(4-methoxystyryl) quinoxaline 1,4-dioxide (11d)

Orange solid; yield $0.10 \mathrm{~g}(25 \%)$; mp $214-215{ }^{\circ} \mathrm{C}$; IR (KBr) v / cm ${ }^{-1} 3072(\mathrm{Ar}-\mathrm{H}), 2954(\mathrm{C}-\mathrm{H}$ saturated), $1738(\mathrm{C}=\mathrm{O}$ ester $), 1602(\mathrm{C}=\mathrm{C}$ aliphatic $), 1506(\mathrm{C}=\mathrm{C}$ aromatic), $1338(\mathrm{~N}-\mathrm{O}), 1250(\mathrm{C}-\mathrm{O}), 814(\mathrm{C}-\mathrm{Cl})$; UV (DMSO) $\lambda_{\max } / \mathrm{nm} \mathrm{339;}{ }^{1} \mathrm{H}$ NMR $\left(500 \mathrm{MHz}, \mathrm{CDCl}_{3}\right)$ $\delta 1.49$ (t, $\left.J 7.2 \mathrm{~Hz}, 3 \mathrm{H}, \mathrm{OCH}_{2} \mathrm{CH}_{3}\right), 3.88\left(\mathrm{~s}, 3 \mathrm{H}, \mathrm{OCH}_{3}\right)$, $4.63\left(\mathrm{q}, J 7.2 \mathrm{~Hz}, 2 \mathrm{H}, \mathrm{OCH}_{2} \mathrm{CH}_{3}\right), 6.96\left(\mathrm{~d}, J_{\text {ortho }} 8.8 \mathrm{~Hz}, 2 \mathrm{H}\right.$, $\mathrm{Ar}), 7.11\left(\mathrm{~d}, J_{\text {trans }} 16.0 \mathrm{~Hz}, 1 \mathrm{H}, \mathrm{H}_{\mathrm{b}}\right), 7.54\left(\mathrm{~d}, J_{\text {ortho }} 8.8 \mathrm{~Hz}\right.$, $2 \mathrm{H}, \mathrm{Ar}), 7.83\left(\mathrm{dd}, J_{\text {ortho }} 9.2, J_{W} 2.1 \mathrm{~Hz}, 1 \mathrm{H}, \mathrm{H}_{\mathrm{B}}\right), 8.43(\mathrm{~d}$, $\left.J_{\text {trans }} 16.0 \mathrm{~Hz}, 1 \mathrm{H}, \mathrm{H}_{\mathrm{a}}\right), 8.59\left(\mathrm{~m}, 2 \mathrm{H}, \mathrm{H}_{\mathrm{A}}, \mathrm{H}_{\mathrm{C}}\right) ;{ }^{13} \mathrm{C} \mathrm{NMR}$ $\left(500 \mathrm{MHz}, \mathrm{CDCl}_{3}\right) \delta 14.07,55.44,63.84,111.88,114.55$, $119.85,121.89,128.46,129.54,133.45,135.69,135.99$, 136.90, 137.39, 138.19, 141.43, 159.80, 161.52; MS (m/z) $400(56)[\mathrm{M}]^{+}, 402(18)[\mathrm{M}+2]^{+}, 135$ (100). Anal. calcd. for $\mathrm{C}_{20} \mathrm{H}_{17} \mathrm{ClN}_{2} \mathrm{O}_{5}: \mathrm{C}, 59.93 ; \mathrm{H}, 4.28 ; \mathrm{N}, 6.99$. Found: $\mathrm{C}$, 59.78; H, 4.08; N, 6.91.

(E)-6-Chloro-3-(ethoxycarbonyl)-2-(4-methylstyryl) quinoxaline 1,4-dioxide (11e)

Orange solid; yield $0.12 \mathrm{~g} \mathrm{(32 \% );} \mathrm{mp} \mathrm{202-204}{ }^{\circ} \mathrm{C}$; IR $(\mathrm{KBr}) v / \mathrm{cm}^{-1} 3072(\mathrm{Ar}-\mathrm{H}), 2978$ (C-H saturated), 1739 $(\mathrm{C}=\mathrm{O}$ ester), 1599 ( $\mathrm{C}=\mathrm{C}$ aliphatic), $1502(\mathrm{C}=\mathrm{C}$ aromatic), $1356(\mathrm{~N}-\mathrm{O}), 1236(\mathrm{C}-\mathrm{O}), 802(\mathrm{C}-\mathrm{Cl})$; UV (DMSO) $\lambda_{\max } / \mathrm{nm} 333 ;{ }^{1} \mathrm{H}$ NMR $\left(500 \mathrm{MHz}, \mathrm{CDCl}_{3}\right) \delta 1.44(\mathrm{t}$, $\left.J 7.2 \mathrm{~Hz}, 3 \mathrm{H}, \mathrm{OCH}_{2} \mathrm{CH}_{3}\right), 2.36$ (s, 3H, Ar- $\left.\mathrm{CH}_{3}\right), 4.58$ $\left(\mathrm{q}, J 7.2 \mathrm{~Hz}, 2 \mathrm{H}, \mathrm{OCH}_{2} \mathrm{CH}_{3}\right), 7.00\left(\mathrm{~d}, J_{\text {trans }} 16.1 \mathrm{~Hz}, 1 \mathrm{H}\right.$, $\left.\mathrm{H}_{\mathrm{b}}\right), 7.18\left(\mathrm{~d}, J_{\text {ortho }} 7.5 \mathrm{~Hz}, 2 \mathrm{H}, \mathrm{Ar}\right), 7.42\left(\mathrm{~d}, J_{\text {ortho }} 7.5 \mathrm{~Hz}\right.$, $2 \mathrm{H}, \mathrm{Ar}), 7.78\left(\mathrm{dd}, J_{\text {ortho }} 9.2, J_{W} 2.2 \mathrm{~Hz}, 1 \mathrm{H}, \mathrm{H}_{\mathrm{B}}\right), 8.32(\mathrm{~d}$,
$\left.J_{\text {trans }} 16.1 \mathrm{~Hz}, 1 \mathrm{H}, \mathrm{H}_{\mathrm{a}}\right), 8.54\left(\mathrm{~d}, 2 \mathrm{H}, \mathrm{H}_{\mathrm{A}}, \mathrm{H}_{\mathrm{C}}\right) ;{ }^{13} \mathrm{C} \mathrm{NMR}$ $\left(500 \mathrm{MHz}, \mathrm{CDCl}_{3}\right) \delta 14.07,21.47,63.87,113.71,118.31$, 199.79, 121.88, 127.78, 129.73, 132.85, 133.46, 136.02, 136.80, 137.28, 138.34, 140.81, 141.54, 159.67; MS ( $/ \mathrm{z} / \mathrm{z})$ 384 (12) [M] $]^{+}, 386(14)[\mathrm{M}+2]^{+}, 119$ (100). Anal. calcd. for $\mathrm{C}_{20} \mathrm{H}_{17} \mathrm{ClN}_{2} \mathrm{O}_{4}: \mathrm{C}, 62.42 ; \mathrm{H}, 4.45 ; \mathrm{N}, 7.28$. Found: $\mathrm{C}$, $62.50 ; \mathrm{H}, 4.22 ; \mathrm{N}, 7.38$.

(E)-2-(4-Bromostyryl)-6-chloro-3-(ethoxycarbonyl) quinoxaline 1,4-dioxide (11f)

Orange solid; yield $0.10 \mathrm{~g}(23 \%)$; mp $183-185^{\circ} \mathrm{C}$; IR $(\mathrm{KBr}) \mathrm{v} / \mathrm{cm}^{-1} 3097(\mathrm{Ar}-\mathrm{H}), 2925$ (C-H saturated), 1734 $(\mathrm{C}=\mathrm{O}$ ester $), 1610(\mathrm{C}=\mathrm{C}$ aliphatic $), 1540(\mathrm{C}=\mathrm{C}$ aromatic $)$, 1354 (N-O), 1236 (C-O), 804 (C-Cl); UV (DMSO) $\lambda_{\max } / \mathrm{nm} \mathrm{333;}{ }^{1} \mathrm{H}$ NMR $\left(500 \mathrm{MHz}, \mathrm{CDCl}_{3}\right) \delta 1.43(\mathrm{t}$, $\left.J 7.2 \mathrm{~Hz}, 3 \mathrm{H}, \mathrm{OCH}_{2} \mathrm{CH}_{3}\right), 4.58\left(\mathrm{q}, J 7.2 \mathrm{~Hz}, 2 \mathrm{H}, \mathrm{OCH}_{2} \mathrm{CH}_{3}\right)$, $7.03\left(\mathrm{~d}, J_{\text {trans }} 16.1 \mathrm{~Hz}, 1 \mathrm{H}, \mathrm{H}_{\mathrm{b}}\right), 7.37\left(\mathrm{~d}, J_{\text {ortho }} 8.6 \mathrm{~Hz}, 2 \mathrm{H}\right.$, Ar), $7.53\left(\mathrm{~d}, J_{\text {ortho }} 8.6 \mathrm{~Hz}, 2 \mathrm{H}, \mathrm{Ar}\right), 7.80$ (dd, $J_{\text {ortho }} 9.3$, $\left.J_{W} 2.4 \mathrm{~Hz}, 1 \mathrm{H}, \mathrm{H}_{\mathrm{B}}\right), 8.30\left(\mathrm{~d}, J_{\text {trans }} 16.1 \mathrm{~Hz}, 1 \mathrm{H}, \mathrm{H}_{\mathrm{a}}\right), 8.55(\mathrm{~m}$, $\left.2 \mathrm{H}, \mathrm{H}_{\mathrm{A}}, \mathrm{H}_{\mathrm{C}}\right) ;{ }^{13} \mathrm{C} \mathrm{NMR}\left(500 \mathrm{MHz}, \mathrm{CDCl}_{3}\right) \delta 14.05,63.99$, $114.83,119.88,121.95,129.12,129.21,131.11,132.16$, $132.28,133.65,134.54,136.83,137.93,138.73,140.14$, 159.61; MS (m/z) $448(22)[\mathrm{M}]^{+}, 185$ (100). Anal. calcd. for $\mathrm{C}_{19} \mathrm{H}_{14} \mathrm{BrClN}_{2} \mathrm{O}_{4}$ : C, 50.75; H, 3.14; N, 6.23. Found: C, $50.55 ; \mathrm{H}, 3.10 ; \mathrm{N}, 6.11$.

(E)-6-Chloro-2-(3-nitrostyryl)quinoxaline 1,4-dioxide (12a)

It was washed with boiling ethanol and obtained as a fluffy yellow solid; yield $0.10 \mathrm{~g}(29 \%)$; mp 242-244 ${ }^{\circ} \mathrm{C}$; IR $(\mathrm{KBr}) \mathrm{v} / \mathrm{cm}^{-1} 3070(\mathrm{Ar}-\mathrm{H}), 1599$ (C=C aliphatic), 1531 $(\mathrm{C}=\mathrm{C}$ aromatic), $1367(\mathrm{~N}-\mathrm{O}), 767$ (C-Cl); UV (DMSO) $\lambda_{\max } / \mathrm{nm} 326 ;{ }^{1} \mathrm{H}$ NMR (500 MHz, DMSO- $\left.d_{6}\right) \delta 7.71(\mathrm{~d}$, $\left.J_{\text {trans }} 16.5 \mathrm{~Hz}, 1 \mathrm{H}, \mathrm{H}_{\mathrm{a}}\right), 7.80(\mathrm{~d}, J 9.2 \mathrm{~Hz}, 1 \mathrm{H}, \mathrm{H} 6), 8.01(\mathrm{dd}$, $\left.J_{\text {ortho }} 9.2, J_{W} 2.3 \mathrm{~Hz}, 1 \mathrm{H}, \mathrm{H}_{\mathrm{B}}\right), 8.18\left(\mathrm{~d}, J_{\text {ortho }} 8.0 \mathrm{~Hz}, 1 \mathrm{H}, \mathrm{H} 4\right)$, $8.24\left(\mathrm{dd}, J_{\text {ortho }} 8.0, J_{W} 2.3 \mathrm{~Hz}, 1 \mathrm{H}, \mathrm{H} 2\right), 8.37$ (d, $J_{\text {trans }} 16.5 \mathrm{~Hz}$, $\left.1 \mathrm{H}, \mathrm{H}_{\mathrm{b}}\right), 8.44\left(\mathrm{~d}, J_{W} 2.3 \mathrm{~Hz}, 1 \mathrm{H}, \mathrm{H}_{\mathrm{A}}\right), 8.50(\mathrm{~m}, 2 \mathrm{H}, \mathrm{H} 3$, $\left.\mathrm{H}_{\mathrm{C}}\right), 9.20\left(\mathrm{~s}, 1 \mathrm{H}, \mathrm{H}_{\mathrm{D}}\right) ;{ }^{13} \mathrm{C}$ NMR $\left(500 \mathrm{MHz}\right.$, DMSO- $\left.d_{6}\right) \delta$ 118.39, 119.11, 121.97, 122.35, 130.61, 130.67, 132.60, 133.59, 135.89, 136.39, 137.08, 137.22, 137.56, 139.37, 148.34; MS (m/z) $343(99)[\mathrm{M}]^{+}, 345(29)[\mathrm{M}+2]^{+}, 327$ (100). Anal. calcd. for $\mathrm{C}_{16} \mathrm{H}_{10} \mathrm{ClN}_{3} \mathrm{O}_{4}: \mathrm{C}, 55.91 ; \mathrm{H}, 2.93$; $\mathrm{N}, 12.23$. Found: C, 56.11; H, 2.70; N, 12.56.

(E)-6-Chloro-2-(2-hydroxystyryl)quinoxaline 1,4-dioxide (12b)

It was washed with boiling methylene chloride and obtained as a yellow solid; yield $0.10 \mathrm{~g}(32 \%)$; mp 252-254 ${ }^{\circ} \mathrm{C}$; IR (KBr) v / $\mathrm{cm}^{-1} 3410(\mathrm{O}-\mathrm{H}), 3075$ (Ar-H), 1599 ( $\mathrm{C}=\mathrm{C}$ aliphatic), 1525 ( $\mathrm{C}=\mathrm{C}$ aromatic), 1369 (N-O), 764 (C-Cl); UV (DMSO) $\lambda_{\max } / \mathrm{nm} 320 ;{ }^{1} \mathrm{H}$ NMR $\left(500 \mathrm{MHz}, \mathrm{DMSO}-d_{6}\right) \delta 6.87(\mathrm{t}, J 8.0 \mathrm{~Hz}, 1 \mathrm{H}, \mathrm{Ar}), 6.94$ 
(d, $\left.J_{\text {ortho }} 8.1 \mathrm{~Hz}, 1 \mathrm{H}, \mathrm{Ar}\right), 7.22$ (t, J $\left.8.0 \mathrm{~Hz}, 1 \mathrm{H}, \mathrm{Ar}\right), 7.57$ (d, $\left.J_{\text {orho }} 8.1 \mathrm{~Hz}, 1 \mathrm{H}, \mathrm{Ar}\right), 7.73\left(\mathrm{~d}, J_{\text {trans }} 16.7 \mathrm{~Hz}, 1 \mathrm{H}, \mathrm{H}_{\mathrm{a}}\right.$ ), $7.98\left(\mathrm{~d}, J_{\text {ortho }} 9.2 \mathrm{~Hz}, 1 \mathrm{H}, \mathrm{H}_{\mathrm{B}}\right), 8.30$ (d, $J_{\text {trans }} 16.7 \mathrm{~Hz}, 1 \mathrm{H}$, $\left.\mathrm{H}_{\mathrm{b}}\right), 8.42\left(\mathrm{~s}, 1 \mathrm{H}, \mathrm{H}_{\mathrm{A}}\right), 8.47\left(\mathrm{~d}, J_{\text {ortho }} 9.2 \mathrm{~Hz}, 1 \mathrm{H}, \mathrm{H}_{\mathrm{C}}\right), 9.16$ $\left(\mathrm{s}, 1 \mathrm{H}, \mathrm{H}_{\mathrm{D}}\right), 10.42\left(\mathrm{~s}, 1 \mathrm{H}\right.$, exchanges with $\left.\mathrm{D}_{2} \mathrm{O}, \mathrm{Ar}-\mathrm{O}-\underline{\mathrm{H}}\right)$; ${ }^{13} \mathrm{C}$ NMR $\left(500 \mathrm{MHz}\right.$, DMSO- $\left.d_{6}\right) \delta 116.03,116.62,119.44$, $120.29,122.63,122.92,129.62,130.89,131.72,133.18$, 135.64, 136.62, 136.93, 137.41, 141.11, 156.88; MS (m/z) 314 (33) $[\mathrm{M}]^{+}, 60$ (100). Anal. calcd. for $\mathrm{C}_{16} \mathrm{H}_{11} \mathrm{ClN}_{2} \mathrm{O}_{3}: \mathrm{C}$, $61.06 ; \mathrm{H}, 3.52 ; \mathrm{N}, 8.90$. Found: C, 60.90; H, 3.59; N, 8.98.

Synthesis of 6-chloro-3-(hydrazinecarbonyl)-2-methylquinoxaline 1,4-dioxide (13)

A solution of 6-chloro-3-(ethoxycarbonyl)-2-methylquinoxaline 1,4-dioxide (7, $1.13 \mathrm{~g}, 4 \mathrm{mmol})$, hydrazine hydrate $(2 \mathrm{~g}, 40 \mathrm{mmol})$ and ethanol $(20 \mathrm{~mL})$ was refluxed on a steam bath for an hour. Then the product was filtered off and recrystallized from ethanol as pale yellow needlelike crystals. Yield $0.60 \mathrm{~g}(56 \%)$; $\mathrm{mp} 242-244{ }^{\circ} \mathrm{C}$; IR (KBr) $v / \mathrm{cm}^{-1} \mathrm{NH}_{2}$ 3352, 3307, NH 3199, 3062 (Ar-H), 2962 (C-H saturated), $1674(\mathrm{C}=\mathrm{O}$ hydrazide $), 1570(\mathrm{C}=\mathrm{C}$ aromatic), $1327(\mathrm{~N}-\mathrm{O}), 1273(\mathrm{C}-\mathrm{O}), 648(\mathrm{C}-\mathrm{Cl})$; UV (DMSO) $\lambda_{\max } / \mathrm{nm} \mathrm{326;}{ }^{1} \mathrm{H}$ NMR (500 MHz, DMSO- $d_{6}$ ) $\delta 2.59\left(\mathrm{~s}, 3 \mathrm{H}, \mathrm{CH}_{3}\right), 4.71\left(\mathrm{~s}, 2 \mathrm{H}\right.$, exchanges with $\left.\mathrm{D}_{2} \mathrm{O}, \mathrm{NH}_{2}\right)$, $7.91\left(\mathrm{dd}, J_{\text {ortho }} 9.2, J_{W} 2.3 \mathrm{~Hz}, 1 \mathrm{H}, \mathrm{H}_{\mathrm{B}}\right), 8.23\left(\mathrm{~d}, J_{W} 2.3 \mathrm{~Hz}\right.$, $\left.1 \mathrm{H}, \mathrm{H}_{\mathrm{A}}\right), 8.46\left(\mathrm{~d}, J_{\text {ortho }} 9.2 \mathrm{~Hz}, 1 \mathrm{H}, \mathrm{H}_{\mathrm{C}}\right), 9.97(\mathrm{~s}, 1 \mathrm{H}$, exchanges with $\left.\mathrm{D}_{2} \mathrm{O}, \mathrm{NH}\right) ;{ }^{13} \mathrm{C}$ NMR (500 MHz, DMSO- $d_{6}$ ) $\delta$ 14.02, 120.71, 128.83, 131.90, 135.30, 136.31, 139.72, 142.66, 151.52, 163.80; MS (m/z) 268 (19) [M] ${ }^{+}, 151$ (100). Anal. calcd. for $\mathrm{C}_{10} \mathrm{H}_{9} \mathrm{ClN}_{4} \mathrm{O}_{3}$ : C, 44.71; $\mathrm{H}, 3.38 ; \mathrm{N}, 20.85$. Found: C, 44.63; H, 3.67; N, 20.82.

Synthesis of 6-chloro-2-methyl-3-(5-thioxo-4,5-dihydro1,3,4-oxadiazol-2-yl)quinoxaline 1,4-dioxide (14)

A solution of 6-chloro-3-(hydrazinecarbonyl)2-methylquinoxaline 1,4-dioxide (13,0.54 g, 2 mmol), potassium hydroxide $(0.11 \mathrm{~g}, 2 \mathrm{mmol})$, excess carbon disulfide $(2 \mathrm{~mL})$ and ethanol $(20 \mathrm{~mL})$ was refluxed for $24 \mathrm{~h}$. The resulting solution was acidified by $\mathrm{HCl}$ solution $(10 \mathrm{~mL}, 1 \mathrm{M})$, the formed precipitate was filtered off, washed with $\mathrm{HCl}(10 \mathrm{~mL}, 0.1 \mathrm{M})$ and recrystalized from ethanol as yellow crystals. Yield $0.42 \mathrm{~g}(68 \%)$; mp 252-256 ${ }^{\circ} \mathrm{C}$; IR (KBr) v / cm-1 $3136(\mathrm{NH}), 3047(\mathrm{Ar}-\mathrm{H}), 2947(\mathrm{C}-\mathrm{H}$ saturated), $1570(\mathrm{C}=\mathrm{C}$ aromatic), $1342(\mathrm{~N}-\mathrm{O}), 1307(\mathrm{C}=\mathrm{S})$, 1256 (C-O), 833 (C-Cl); UV (DMSO) $\lambda_{\max } / \mathrm{nm} 336$; ${ }^{1} \mathrm{H}$ NMR $\left(500 \mathrm{MHz}, \mathrm{DMSO}-d_{6}\right) \delta 2.84\left(\mathrm{~s}, 3 \mathrm{H}, \mathrm{CH}_{3}\right), 7.95$ (dd, $\left.J_{\text {ortho }} 9.2, J_{W} 2.3 \mathrm{~Hz}, 1 \mathrm{H}, \mathrm{H}_{\mathrm{B}}\right), 8.41\left(\mathrm{~d}, J_{W} 2.3 \mathrm{~Hz}, 1 \mathrm{H}\right.$, $\left.\mathrm{H}_{\mathrm{A}}\right), 8.46\left(\mathrm{~d}, J_{\text {ortho }} 9.2 \mathrm{~Hz}, 1 \mathrm{H}, \mathrm{H}_{\mathrm{C}}\right), 9.97(\mathrm{~s}, 1 \mathrm{H}$, exchanges with $\mathrm{D}_{2} \mathrm{O}, \mathrm{NH}$ ); ${ }^{13} \mathrm{C}$ NMR (500 MHz, DMSO- $\left.d_{6}\right) \delta 14.09$,
$120.58,128.93,132.45,135.06,136.45,140.31,140.79$, 142.50, 157.84, 177.70; MS (m/z): $310(33)[\mathrm{M}]^{+}, 312$ (61) $[\mathrm{M}+2]^{+}, 55$ (100). Anal. calcd. for $\mathrm{C}_{11} \mathrm{H}_{7} \mathrm{ClN}_{4} \mathrm{O}_{3} \mathrm{~S}: \mathrm{C}, 42.52$; H, 2.27; N, 18.03. Found: C, 42.49; H, 2.38; N, 18.35 .

Synthesis of 6-chloro-2-methyl-3-(5-(4-nitrophenyl)1,3,4-oxadiazol-2-yl)quinoxaline 1,4-dioxide (15)

A mixture of 6-chloro-3-(hydrazinecarbonyl)2-methylquinoxaline 1,4-dioxide $\mathbf{1 3}(0.27 \mathrm{~g}, 1 \mathrm{mmol})$, p-nitrobenzoic acid $(0.17 \mathrm{~g}, 1 \mathrm{mmol})$ and phosphorus oxychloride $(2 \mathrm{~mL})$ was heated under reflux for $2 \mathrm{~h}$ at $70^{\circ} \mathrm{C}$ in water bath then the produced solution was poured into ice-water. The formed precipitate was filtered off and recrystallized from ethanol-water as a gray powder. Yield $0.20 \mathrm{~g}(50 \%)$; mp $96-97{ }^{\circ} \mathrm{C}$; IR $(\mathrm{KBr}) \mathrm{v} / \mathrm{cm}^{-1}$ $3079(\mathrm{Ar}-\mathrm{H}), 2990(\mathrm{C}-\mathrm{H}$ saturated $), 1602(\mathrm{C}=\mathrm{N}), 1527$ $(\mathrm{C}=\mathrm{C}$ aromatic), $1346(\mathrm{~N}-\mathrm{O}), 1275(\mathrm{C}-\mathrm{O}), 717(\mathrm{C}-\mathrm{Cl})$; ${ }^{1} \mathrm{H}$ NMR $\left(500 \mathrm{MHz}, \mathrm{CDCl}_{3}\right) \delta 1.77\left(\mathrm{~s}, 3 \mathrm{H}, \mathrm{CH}_{3}\right), 8.07$ (d, $\left.J_{\text {ortho }} 8.4 \mathrm{~Hz}, 2 \mathrm{H}, \mathrm{Ar}\right), 8.14\left(\mathrm{~d}, J_{\text {ortho }} 8.1 \mathrm{~Hz}, 1 \mathrm{H}, \mathrm{H}_{\mathrm{C}}\right.$ ), 8.25-8.34 (m, 4H, $\mathrm{H}_{\mathrm{A}}, \mathrm{H}_{\mathrm{B}}, 2$ for $\left.\mathrm{Ar}\right) ;{ }^{13} \mathrm{C} \mathrm{NMR}(500 \mathrm{MHz}$, $\left.\mathrm{CDCl}_{3}\right) \delta 24.08,44.75,65.65,123.62,124.10,124.52$, 128.08, 128.61, 130.56, 131.02, 133.91, 140.27, 141.42, 150.05, 164.59; MS (m/z) 399 (3) [M] $]^{+}, 312$ (100). Anal. calcd. for $\mathrm{C}_{17} \mathrm{H}_{10} \mathrm{ClN}_{5} \mathrm{O}_{5}$ : C, 51.08; H, 2.52; N, 17.52. Found: C, 51.28; H, 2.59; N, 17.83 .

Synthesis of $(E)$-6-chloro-2-methyl-3-(2-(4-substitutedbenzylidene)hydrazine-1-carbonyl)quinoxaline 1,4-dioxide derivatives (16a-e)

A solution of 6-chloro-3-(hydrazinecarbonyl)2-methylquinoxaline 1,4-dioxide (13, $0.27 \mathrm{~g}, 1 \mathrm{mmol})$, the crossponding aromatic aldehyde $(1 \mathrm{mmol}), 3$ drops of glacial acetic acid and ethanol $(10 \mathrm{~mL})$ was refluxed for 8-12 h. The produced precipitate was filtered off and recrystallized from the proper solvent.

(E)-3-(2-Benzylidenehydrazine-1-carbonyl)-6-chloro2-methylquinoxaline 1,4-dioxide (16a)

Yellow crystals obtained after washing with boiling ethanol. Yield $0.34 \mathrm{~g}(95 \%)$; mp 256-258 ${ }^{\circ} \mathrm{C}$; IR (KBr) $v / \mathrm{cm}^{-1} 3315(\mathrm{NH}), 3101$ (Ar-H), 2990 (C-H saturated), $1660(\mathrm{C}=\mathrm{O}$ hydrazide $), 1600(\mathrm{C}=\mathrm{N}), 1550(\mathrm{C}=\mathrm{C}$ aromatic $)$, $1334(\mathrm{~N}-\mathrm{O}), 1118(\mathrm{C}-\mathrm{O}), 717(\mathrm{C}-\mathrm{Cl})$; UV (DMSO) $\lambda_{\max } / \mathrm{nm} 340 ;{ }^{1} \mathrm{H}$ NMR (500 MHz, DMSO- $\left.d_{6}\right) \delta 2.68(\mathrm{~s}, 3 \mathrm{H}$, $\left.\mathrm{CH}_{3}\right), 7.25-7.53(\mathrm{~m}, 5 \mathrm{H}, \mathrm{Ar}), 7.95\left(\mathrm{~d}, J_{\text {ortho }} 8.6 \mathrm{~Hz}, 1 \mathrm{H}, \mathrm{H}_{\mathrm{C}}\right.$ ), $8.32\left(\mathrm{~s}, 1 \mathrm{H}, \mathrm{H}_{\mathrm{D}}\right), 8.45-8.54\left(\mathrm{~m}, 2 \mathrm{H}, \mathrm{H}_{\mathrm{A}}, \mathrm{H}_{\mathrm{B}}\right), 12.30(\mathrm{~s}, 1 \mathrm{H}$, exchanges with $\left.\mathrm{D}_{2} \mathrm{O}, \mathrm{NH}\right) ;{ }^{13} \mathrm{C}$ NMR (500 MHz, DMSO- $d_{6}$ ) $\delta 14.01,120.82,127.08,127.73,128.95,129.17,129.26$, 130.98, 132.30, 134.00, 135.64, 136.48, 140.34, 142.47, 
150.16, 150.26, 160.87; MS (m/z) 356 (34) [M] $]^{+}, 306(100)$. Anal. calcd. for $\mathrm{C}_{17} \mathrm{H}_{13} \mathrm{ClN}_{4} \mathrm{O}_{3}$ : C, 57.23; $\mathrm{H}, 3.67 ; \mathrm{N}, 15.70$. Found: C, 57.22; H, 3.72; N, 15.66.

(E)-6-Chloro-3-(2-(4-methoxybenzylidene)hydrazine1-carbonyl)-2-methylquinoxaline 1,4-dioxide (16b)

Bright yellow plates (ethanol); yield $0.34 \mathrm{~g}(89 \%) ; \mathrm{mp}$ 245-250 ${ }^{\circ} \mathrm{C}$; IR ( $\left.\mathrm{KBr}\right) \vee / \mathrm{cm}^{-1} 3305(\mathrm{NH}), 3074(\mathrm{Ar}-\mathrm{H})$, $2980(\mathrm{C}-\mathrm{H}$ saturated $), 1689(\mathrm{C}=\mathrm{O}$ hydrazide $), 1604(\mathrm{C}=\mathrm{N})$, $1500(\mathrm{C}=\mathrm{C}$ aromatic $), 1336(\mathrm{~N}-\mathrm{O}), 1101(\mathrm{C}-\mathrm{O}), 829$ (C-Cl); UV (DMSO) $\lambda_{\max } / \mathrm{nm} \mathrm{340;}{ }^{1} \mathrm{H}$ NMR $(500 \mathrm{MHz}$, DMSO- $\left.d_{6}\right) \delta 2.67\left(\mathrm{~s}, 3 \mathrm{H}, \mathrm{CH}_{3}\right), 3.82\left(\mathrm{~s}, 3 \mathrm{H}, \mathrm{OCH}_{3}\right), 7.04$ (d, $\left.J_{\text {ortho }} 8.6 \mathrm{~Hz}, 2 \mathrm{H}, \mathrm{Ar}\right), 7.71$ (d, $\left.J_{\text {ortho }} 8.6 \mathrm{~Hz}, 2 \mathrm{H}, \mathrm{Ar}\right), 7.95$ (dd, $\left.J_{\text {ortho }} 9.2, J_{W} 2.3 \mathrm{~Hz}, 1 \mathrm{H}, \mathrm{H}_{\mathrm{B}}\right), 8.31\left(\mathrm{~d}, J_{W} 2.3 \mathrm{~Hz}, 1 \mathrm{H}\right.$, $\left.\mathrm{H}_{\mathrm{A}}\right), 8.39\left(\mathrm{~s}, 1 \mathrm{H}, \mathrm{H}_{\mathrm{D}}\right), 8.49\left(\mathrm{~d}, J 9.2 \mathrm{~Hz}, 1 \mathrm{H}, \mathrm{H}_{\mathrm{C}}\right), 12.16$ (s, $1 \mathrm{H}$, exchanges with $\left.\mathrm{D}_{2} \mathrm{O}, \mathrm{NH}\right) ;{ }^{13} \mathrm{C}$ NMR $(500 \mathrm{MHz}$, DMSO- $\left.d_{6}\right) \delta 13.93,55.55,114.53,114.62,120.72,126.50$, $128.83,129.33,132.11,135.50,136.33,140.16,142.41$, 149.85, 150.44, 161.43; MS (m/z) $386(19)[\mathrm{M}]^{+}, 370$ (100). Anal. calcd. for $\mathrm{C}_{18} \mathrm{H}_{15} \mathrm{ClN}_{4} \mathrm{O}_{4}$ : C, 55.90; H, 3.91; N, 14.49. Found: C, 55.58; H, 4.11; N, 14.42 .

(E)-6-Chloro-2-methyl-3-(2-(4-nitrobenzylidene)hydrazine1-carbonyl)quinoxaline 1,4-dioxide (16c)

Yellow crystals obtained after washing with boiling ethanol. Yield $0.39 \mathrm{~g}(97 \%) ; \mathrm{mp} 272-274{ }^{\circ} \mathrm{C}$; IR (KBr) $\mathrm{v} / \mathrm{cm}^{-1} 3347(\mathrm{NH}), 3083(\mathrm{Ar}-\mathrm{H}), 2960$ (C-H saturated), $1680(\mathrm{C}=\mathrm{O}$ hydrazide $), 1593(\mathrm{C}=\mathrm{N}), 1533(\mathrm{C}=\mathrm{C}$ aromatic $)$, $1342(\mathrm{~N}-\mathrm{O}), 1112(\mathrm{C}-\mathrm{O}), 835(\mathrm{C}-\mathrm{Cl})$; UV (DMSO) $\lambda_{\max } / \mathrm{nm} 340 ;{ }^{1} \mathrm{H}$ NMR (500 MHz, DMSO- $\left.d_{6}\right) \delta 2.69$ (s, $\left.3 \mathrm{H}, \mathrm{CH}_{3}\right), 8.03$ (d, $\left.J_{\text {ortho }} 8.8 \mathrm{~Hz}, 2 \mathrm{H}, \mathrm{Ar}\right), 8.13$ (d, $J_{\text {ortho }} 9.1$, $\left.J_{W} 2.3 \mathrm{~Hz}, 1 \mathrm{H}, \mathrm{H}_{\mathrm{B}}\right), 8.28-8.35\left(\mathrm{~m}, 3 \mathrm{H}, \mathrm{H}_{\mathrm{A}}, 2\right.$ for $\left.\mathrm{Ar}\right), 8.50$ $\left(\mathrm{d}, J_{\text {ortho }} 9.1 \mathrm{~Hz}, 1 \mathrm{H}, \mathrm{H}_{\mathrm{C}}\right), 8.58\left(\mathrm{~s}, 1 \mathrm{H}, \mathrm{H}_{\mathrm{D}}\right), 12.10(\mathrm{~s}, 1 \mathrm{H}$, exchanges with $\left.\mathrm{D}_{2} \mathrm{O}, \mathrm{NH}\right) ;{ }^{13} \mathrm{C}$ NMR $\left(500 \mathrm{MHz}\right.$, DMSO- $d_{6}$ ) $\delta 13.97,120.83,124.40,128.15,128.67,128.93,132.40$, 135.70, 136.49, 140.29, 140.50, 142.38, 147.71, 148.47, 161.14; MS (m/z) $401(17)[\mathrm{M}]^{+}, 177$ (100). Anal. calcd. for $\mathrm{C}_{17} \mathrm{H}_{12} \mathrm{ClN}_{5} \mathrm{O}_{5}: \mathrm{C}, 50.82 ; \mathrm{H}, 3.01 ; \mathrm{N}, 17.43$. Found: $\mathrm{C}$, $50.98 ; \mathrm{H}, 2.99 ; \mathrm{N}, 17.75$.

(E)-3-(2-(4-Bromobenzylidene)hydrazine-1-carbonyl)6-chloro-2-methylquinoxaline 1,4-dioxide (16d)

Yellow crystals obtained after washing with boiling ethanol. Yield $0.40 \mathrm{~g}(92 \%) ; \mathrm{mp} 276-278{ }^{\circ} \mathrm{C}$; IR (KBr) $\mathrm{v} / \mathrm{cm}^{-1} 3241(\mathrm{NH}), 3099(\mathrm{Ar}-\mathrm{H}), 2919(\mathrm{C}-\mathrm{H}$ saturated), $1687(\mathrm{C}=\mathrm{O}$ hydrazide $), 1595(\mathrm{C}=\mathrm{N}), 1568(\mathrm{C}=\mathrm{C}$ aromatic $)$, $1338(\mathrm{~N}-\mathrm{O}), 1115(\mathrm{C}-\mathrm{O}), 825(\mathrm{C}-\mathrm{Cl})$; UV (DMSO) $\lambda_{\max } / \mathrm{nm} 339 ;{ }^{1} \mathrm{H}$ NMR (500 MHz, DMSO- $d_{6}$ ) $\delta 2.67$ (s, 3H, $\mathrm{CH}_{3}$ ), 7.66-7.75 (m, 4H, Ar), 7.95 (dd, $J_{\text {ortho }} 9.1$, $\left.J_{W} 2.3 \mathrm{~Hz}, 1 \mathrm{H}, \mathrm{H}_{\mathrm{B}}\right), 8.31\left(\mathrm{~d}, J_{W} 2.3,1 \mathrm{H}, \mathrm{H}_{\mathrm{A}}\right), 8.42(\mathrm{~s}, 1 \mathrm{H}$, $\left.\mathrm{H}_{\mathrm{D}}\right), 8.49\left(\mathrm{~d}, J_{\text {ortho }} 9.1 \mathrm{~Hz}, 1 \mathrm{H}, \mathrm{H}_{\mathrm{C}}\right), 12.42(\mathrm{~s}, 1 \mathrm{H}$, exchanges with $\left.\mathrm{D}_{2} \mathrm{O}, \mathrm{NH}\right) ;{ }^{13} \mathrm{C}$ NMR (500 MHz, DMSO- $\left.d_{6}\right) \delta 13.83$, $120.65,124.03,128.73,129.39,132.05,132.56,133.15$, 135.46, 136.26, 140.15, 142.26, 148.72, 149.99, 160.68; MS $(m / z) 435(9)[M+1]^{+}, 420$ (100). Anal. calcd. for $\mathrm{C}_{17} \mathrm{H}_{12} \mathrm{BrClN}_{4} \mathrm{O}_{3}: \mathrm{C}, 46.87 ; \mathrm{H}, 2.78 ; \mathrm{N}, 12.86$. Found: $\mathrm{C}$, 46.96; H, 2.70; N, 12.54 .

(E)-6-Chloro-3-(2-(4-(dimethylamino)benzylidene) hydrazine-1-carbonyl)-2-methylquinoxaline 1,4-dioxide (16e)

Orange crystals (ethanol); yield $0.35 \mathrm{~g}(88 \%)$; mp 245-249 ${ }^{\circ} \mathrm{C}$; IR (KBr) v / cm 3209 (NH), 3091 (Ar-H), 2912 (C-H saturated), $1680(\mathrm{C}=\mathrm{O}$ hydrazide), 1606 $(\mathrm{C}=\mathrm{N}), 1522(\mathrm{C}=\mathrm{C}$ aromatic), $1330(\mathrm{~N}-\mathrm{O}), 1112(\mathrm{C}-\mathrm{O}), 820$ (C-Cl); UV (DMSO) $\lambda_{\max } / \mathrm{nm} 340 ;{ }^{1} \mathrm{H}$ NMR $(500 \mathrm{MHz}$, DMSO- $\left.d_{6}\right) \delta 2.65\left(\mathrm{~s}, 3 \mathrm{H}, \mathrm{CH}_{3}\right), 2.97\left(\mathrm{~s}, 6 \mathrm{H}, \mathrm{N}\left(\mathrm{CH}_{3}\right)_{2}\right), 6.76$ (d, $\left.J_{\text {ortho }} 8.6 \mathrm{~Hz}, 2 \mathrm{H}, \mathrm{Ar}\right), 7.57$ (d, $\left.J_{\text {ortho }} 8.6 \mathrm{~Hz}, 2 \mathrm{H}, \mathrm{Ar}\right), 7.93$ $\left(\mathrm{dd}, J_{\text {ortho }} 9.2, J_{W} 2.3 \mathrm{~Hz}, 1 \mathrm{H}, \mathrm{H}_{\mathrm{B}}\right), 8.25\left(\mathrm{~s}, 1 \mathrm{H}, \mathrm{H}_{\mathrm{D}}\right), 8.29(\mathrm{~d}$, $\left.J_{W} 2.3 \mathrm{~Hz}, 1 \mathrm{H}, \mathrm{H}_{\mathrm{A}}\right), 8.47\left(\mathrm{~d}, J_{\text {ortho }} 9.2 \mathrm{~Hz}, 1 \mathrm{H}, \mathrm{H}_{\mathrm{C}}\right), 11.99$ (s, $1 \mathrm{H}$, exchanges with $\left.\mathrm{D}_{2} \mathrm{O}, \mathrm{NH}\right) ;{ }^{13} \mathrm{C}$ NMR $(500 \mathrm{MHz}$, DMSO- $\left.d_{6}\right) \delta 14.27,112.26,120.95,121.33,128.63,129.15$, 129.41, 131.78, 132.35, 135.77, 136.67, 140.40, 142.77, 151.04, 152.37, 160.55; MS (m/z) 399 (3) [M] ${ }^{+}, 401$ (2) $[\mathrm{M}+2]^{+}, 43$ (100). Anal. calcd. for $\mathrm{C}_{19} \mathrm{H}_{18} \mathrm{ClN}_{5} \mathrm{O}_{3}: \mathrm{C}, 57.08$; H, 4.54; N, 17.52. Found: C, 57.29; H, 4.50; N, 17.42.

\section{Supplementary Information}

Supplementary data are available free of charge at http://jbcs.sbq.org.br as PDF file.

\section{References}

1. Carta, A.; Corona, P.; Loriga, M.; Curr. Med. Chem. 2005, 12, 2259.

2. Liu, J.; Ouyang, M.; Jiang, J.; Mu, P.; Wu, J.; Yang, Q.; Zhang, C.; Xu, W.; Wang, L.; Huen, M. S. Y.; Deng, Y.; Mutat. Res., Genet. Toxicol. Environ. Mutagen. 2012, 741, 70.

3. Vicente, E.; Villar, R.; Pérez-Silanes, S.; Aldana, I.; Goldman, R. C.; Mong, A.; Infect. Disord.: Drug Targets 2011, 11, 196.

4. Zanetti, S.; Sechi, L. A.; Molicotti, P.; Cannas, S.; Carta, A.; Bua, A.; Deriu, A.; Paglietti, G.; Int. J. Antimicrob. Agents 2005, $25,179$.

5. dos Santos Fernandes, G. F.; Moreno-Viguri, E.; SantivañezVeliz, M.; Paucar, R.; Chin, C. M.; Pérez-Silanes, S.; dos Santos, J. L.; J. Heterocycl. Chem. 2017, 54, 2380.

6. Carta, A.; Loriga, M.; Paglietti, G.; Mattana, A.; Fiori, P. L.; Mollicotti, P.; Sechi, L.; Zanetti, S.; Eur. J. Med. Chem. 2004, 39, 195.

7. Lakshmi, Y.; Murthy, N.; Mani, P.; Govindh, B.; Res. J. Pharm., Biol. Chem. Sci. 2011, 2, 553. 
8. Torres, E.; Moreno-Viguri, E.; Galiano, S.; Devarapally, G.; Crawford, P. W.; Azqueta, A.; Arbillaga, L.; Varela, J.; Birriel, E.; Di Maio, R.; Cerecetto, H.; González, M.; Aldana, I.; Monge, A.; Pérez-Silanes, S.; Eur. J. Med. Chem. 2013, 66, 324.

9. Benitez, D.; Cabrera, M.; Hernandez, P.; Boiani, L.; Lavaggi, M. L.; Di Maio, R.; Yaluff, G.; Serna, E.; Torres, S.; Ferreira, M. E.; de Bilbao, N. V.; Torres, E.; Perez-Silanes, S.; Solano, B.; Moreno, E.; Aldana, I.; de Cerain, A. L.; Cerecetto, H.; Gonzalez, M.; Monge, A.; J. Med. Chem. 2011, 54, 3624.

10. Zarranz, B.; Jaso, A.; Aldana, I.; Monge, A.; Maurel, S.; Deharo, E.; Jullian, V.; Sauvain, M.; Drug Res. 2005, 55, 754.

11. Vicente, E.; Charnaud, S.; Bongard, E.; Villar, R.; Burguete, A.; Solano, B.; Ancizu, S.; Pérez-Silanes, S.; Aldana, I.; Vivas, L.; Monge, A.; Molecules 2008, 13, 69.

12. Glazer, E. A.; Chappel, L. R.; J. Med. Chem. 1982, 25, 868.

13. Duque-Montaño, B. E.; Gómez-Caro, L. C.; Sanchez-Sanchez, M.; Monge, A.; Hernández-Baltazar, E.; Rivera, G.; TorresAngeles, O.; Bioorg. Med. Chem. 2013, 21, 4550.

14. Monge, A.; Palop, J. A.; de Cerain, A. L.; Senador, V.; Martinez, F. J.; Sainz, Y.; Narro, S.; Garcia, E.; de Miguel, C.; J. Med. Chem. 1995, 38, 1786.

15. Monge, A.; Martinez-Crespo, F. J.; de Cerain, A. L.; Palop, J. A.; Narro, S.; Senador, V.; Marin, A.; Sainz, Y.; Gonzalez, M.; J. Med. Chem. 1995, 38, 4488.

16. Solano, B.; Junnotula, V.; Marín, A.; Villar, R.; Burguete, A.; Vicente, E.; Pérez-Silanes, S.; Aldana, I.; Monge, A.; Dutta, S.; Sarkar, U.; Gates, K. S.; J. Med. Chem. 2007, 50, 5485.

17. Das, U.; Pati, H. N.; Panda, A. K.; De Clercq, E.; Balzarini, J.; Molnár, J.; Baráth, Z.; Ocsovszki, I.; Kawase, M.; Zhou, L.; Sakagami, H.; Dimmock, J. R.; Bioorg. Med. Chem. 2009, 17, 3909.

18. Burguete, A.; Pontiki, E.; Hadjipavlou-Litina, D.; Villar, R.; Vicente, E.; Solano, B.; Ancizu, S.; Pérez-Silanes, S.; Aldana, I.; Monge, A.; Bioorg. Med. Chem. Lett. 2007, 17, 6439.

19. Burguete, A.; Pontiki, E.; Hadjipavlou-Litina, D.; Ancizu, S.; Villar, R.; Solano, B.; Moreno, E.; Torres, E.; Pérez, S.; Aldana, I.; Monge, A.; Chem. Biol. Drug Des. 2011, 77, 255.
20. Figdor, S. K.; Hobbs, D. C.; Can. J. Chem. 1980, 58, 1957.

21. Loriga, M.; Nuvole, A.; Paglietti, G.; Fadda, G.; Zanetti, S.; Eur. J. Med. Chem. 1990, 25, 527.

22. Lanquist, J. K.; Stacey, G. J.; J. Chem. Soc. 1953, 1, 2822.

23. Haddadin, M. J.; Issidorides, C. H.; Tetrahedron Lett. 1965, 6, 3253.

24. Lia, J.; Jia, M.; Hua, W.; Hua, H.; Indian J. Chem. 2001, 408, 1230.

25. Heyns, K.; Behse, E.; Francke, W.; Eur. J. Inorg. Chem. 1981, $114,240$.

26. Takabatake, T.; Hasegawa, M.; J. Heterocycl. Chem. 1987, 24 , 529.

27. Chupakhin, O. N.; Kotovskaya, S. K.; Perova, N. M.; Baskakova, Z. M.; Charushin, V. N.; Chem. Heterocycl. Compd. 1999, 35, 459.

28. Vega, A. O.; Gil, M. J.; Fernández-Alvarez, E.; J. Heterocycl. Chem. 1984, 21, 1271.

29. Stumm, G.; Niclas, H.-J.; J. Prakt. Chem. 1989, 331, 736.

30. Zarranz, B.; Jaso, A.; Aldana, I.; Monge, A.; Bioorg. Med. Chem. 2003, 11, 2149.

31. Monge, A.; Palop, J. A.; González, M.; Martínez-Crespo, F. J.; de Ceráin, A. L.; Sáinz, Y.; Narro, S.; Barker, A. J.; Hamilton, E.; J. Heterocycl. Chem. 1995, 32, 1213.

32. Gasco, A.; Ermondi, G.; Fruttero, R.; Gasco, A.; Eur. J. Med. Chem. 1996, 31, 3.

33. Jaso, A.; Zarranz, B.; Aldana, I.; Monge, A.; J. Med. Chem. 2005, 48, 2019.

34. Clayden, J.; Greeves, N.; Warren, S. G.; Organic Chemistry, $2^{\text {nd }}$ ed.; Oxford University Press: New York, 2012.

35. Lyubchanskaya, V. M.; Alekseeva, L. M.; Granik, V. G.; Chem. Heterocycl. Compd. 1992, 28, 34.

36. El-Gogary, S. R.; Waly, M. A.; Ibrahim, I. T.; El-Sepelgy, O. Z.; Monatsh. Chem. 2010, 141, 1253.

Submitted: March 13, 2018 Published online: April 16, 2018 